\title{
Colletotrichum orbiculare WHI2, a Yeast Stress-Response Regulator Homolog, Controls the Biotrophic Stage of Hemibiotrophic Infection Through TOR Signaling
}

\author{
Ken Harata, ${ }^{1}$ Takumi Nishiuchi, ${ }^{2}$ and Yasuyuki Kubo ${ }^{1}$ \\ ${ }^{1}$ Laboratory of Plant Pathology, Graduate School of Life and Environmental Sciences, Kyoto Prefectural University, Kyoto, \\ Japan; and ${ }^{2}$ Division of Functional Genomics, Advanced Science Research Centre, Kanazawa University, Kanazawa, Japan \\ Submitted 4 February 2015. Accepted 14 March 2016.
}

\begin{abstract}
The hemibiotrophic fungus Colletotrichum orbiculare first establishes a biotrophic infection stage in cucumber (Cucumber sativus) epidermal cells and subsequently transitions to a necrotrophic stage. Here, we found that $C$. orbiculare established hemibiotrophic infection via $C$. orbiculare WHI2, a yeast stress regulator homolog, and TOR (target of rapamycin) signaling. Plant defense responses such as callose deposition, $\mathrm{H}_{2} \mathrm{O}_{2}$, and antimicrobial proteins were strongly induced by the C. orbiculare whi2s mutant, resulting in defective pathogenesis. Expression analysis of biotrophy-specific genes evaluated by the promoter VENUS fusion gene indicated weaker VENUS signal intensity in the whi2s mutant, thereby suggesting that C. orbiculare WHI2 plays a key role in regulating biotrophic infection of $C$. orbiculare. The involvement of CoWHI2 in biotrophic infection was further explored with a DNA microarray. In the Cowhi2s mutant, TOR-dependent ribosomal proteinrelated genes were strikingly upregulated compared with the wild type. Moreover, callose deposition in the host plant after inoculation with the Cowhi2s mutant treated with rapamycin, which inhibits TOR activity, was reduced, and the mutant remained biotrophic in contrast to the untreated mutant. Thus, regulation of TOR by Whi 2 is apparently crucial to the biotrophic stage of hemibiotrophic infection in $C$. orbiculare.
\end{abstract}

Colletotrichum orbiculare, the causal agent of anthracnose disease on cucurbitaceous plants, establishes infection by developing a series of specialized infection structures (Kubo and Takano 2013). Conidia adhering to the cuticle of host cells germinate and form a melanized dome-like structure called the appressorium that is necessary for successful infection of host plants (Kubo and Furusawa 1991). Recently, we revealed that proper cell cycle regulation during appressorium formation is essential for plant infection (Fukada and Kubo 2015). The infection hyphae emerging from the appressoria of Colletotrichum species initially form bulbous biotrophic hyphae, and the fungus later switches to a necrotrophic phase accompanied by the formation of narrower infection hyphae (Irieda et al. 2014; Perfect et al. 1998).

Nucleotide seqeuence data were deposited in the Gene Expression Omnibus (GEO) database under accession number GSE73096.

Corresponding author: Y. Kubo; E-mail: y_kubo@kpu.ac.jp

*The $\boldsymbol{e}$-Xtra logo stands for "electronic extra" and indicates that eight supplementary figures and three supplementary tables are published online.

(c) 2016 The American Phytopathological Society
The biotrophic stage of hemibiotrophic fungi is a crucial step to obtain nutrients without killing the host cells. Against hemibiotrophic fungal infection, the host plant recognizes fungal-specific molecules such as $\beta$-glucans and chitin, called pathogen-associated molecular patterns (PAMPs), which immediately triggers its defense responses (Fujikawa et al. 2012; Latgé 2010). Therefore, hemibiotrophic fungi must suppress this pattern-triggered immunity caused by recognition of the PAMPs, via pattern recognition receptors to establish the biotrophic stage (Miya et al. 2007; Monaghan and Zipfel 2012).

To circumvent activation of host defense responses, hemibiotrophic fungi secrete effector proteins during the biotrophic stage (Kleemann et al. 2012; Zhang and Xu 2014). In Magnaporthe oryzae, effector protein Slp1 functions to suppress chitin-induced plant immunity (Mentlak et al. 2012). In C. orbiculare, effector proteins Nis1, Dn3, and Mc69 are predominantly expressed and secreted during the biotrophic stage (Saitoh et al. 2012; Yoshino et al. 2012). In addition, many secondary metabolite backbone synthesis genes are upregulated during the biotrophic stage compared with vegetative hyphae, and some genes are specifically expressed during the biotrophic stage in C. orbiculare and $C$. higginsianum (Gan et al. 2013; O'Connell et al. 2012). Thus, appropriate gene expression during the biotrophic stage is envisaged as a key for hemibiotrophic fungi to infect their host plants. However, the regulatory mechanisms involved in hemibiotrophic infection of hemibiotrophic fungi are still not clear.

The target of rapamycin (TOR) is widely conserved among eukaryotes from mammals to yeasts (Brown et al. 1994; Heitman et al. 1991; Kunz et al. 1993; Sabatini et al. 1994). TOR is known as a key factor for pleiotrophic intracellular events such as autophagy, reactive oxygen species, protein synthesis, ribosomal biosynthesis, and metabolism (Alers et al. 2012; Bonawitz et al. 2007; Martin et al. 2006). In plant-pathogenic fungi, TOR is also required for pathogenesis and infection-related morphogenesis. In the hemibiotrophic fungus Fusarium graminearum, the TOR signaling pathway is required for pathogenesis and vegetative hyphae, and serine/threonie kinase SCH9, which functions downstream of the TOR signaling pathway, is involved in conidium size, stress response, and pathogenesis (Chen et al. 2014; Yu et al. 2014). Furthermore, in M. oryzae, TOR activation in reponse to intracellular glutamine levels inhibits appressorium development and TOR activation is required for biotrophic growth inside epidermal cells of rice leaf (Fernandez et al. 2014; Marroquin-Guzman and Wilson 2015). However, how intracellular factors regulate the TOR signaling pathway activity in hemibiotrophic fungi is far less understood. 
In previous reports, $C$. orbiculare small GTP-binding protein CoRas 2 positively regulates activation of the cAMP- protein kinase A (PKA) and the mitogen-activated protein kinase (MAPK) CoMekk1-Cmk1 signaling pathways involved in infection-related morphogenesis and undergo dynamic cellular localization during infection (Harata and Kubo 2014). Therefore, we assumed that the spatiotemporal regulation of CoRas2 has a crucial role in inducing infection-related morphogenesis. In Saccharomyces cerevisiae, the stress response regulator WHI2 contributes to stabilizing F-actin structure in response to nutritional sensing, and F-actin requires the proper localization of the Ras protein (Leadsham et al. 2009). Therefore, to elucidate the relationship between the spatiotemporal regulation of CoRas 2 and infection-related morphogenesis, we functionally analyzed $C$. orbiculare CoWHI2, the homolog of WHI2 of S. cerevisiae.

In phytopathogens, the TOR signaling pathway is known to have pleiotropic roles during the infection process, but the regulatory mechanism for this pathway is far less understood in comparison with the cAMP-PKA and the MAPK signaling pathways required for successful infection. Activity of the transcription factor Msn2p is regulated by $S$. cerevisiae Whi2p and the TOR signaling pathway, but the relation between Whi2p and the TOR signaling pathway is not clearly defined (Sadeh et al. 2011). Therefore, in addition to analyzing the spatiotemporal regulation of CoRas 2 in CoWhi2 function, we also examined the relation between CoWhi2 and the TOR signaling pathway.

In this study, we present evidence for the novel insight that Colletotrichum orbiculare Whi2 controls the highly regulated biotrophic stage of hemibiotrophic infection by $C$. orbiculare via TOR.

\section{RESULTS}

Targeted replacement of CoWHI2 in $C$. orbiculare.

Using $C$. orbiculare genomic information, we searched for a homolog of $S$. cerevisiae WHI2 that could potentially be involved in subcellular localization of the signaling protein Ras, in response to environmental sensing, and found the gene ENH78490, which putatively encodes a protein with 293 amino acids (Gan et al. 2013). The predicted amino acid sequence from ENH78490 showed significant similarity to that of Whi2p in S. cerevisiae (Supplementary Fig. S1). Therefore, we named this gene Colletotrichum orbiculare CoWHI2.

To analyze the function of CoWHI2, we constructed a Cowhi2s disruption vector designed to replace the wild-type CoWHI2 gene with a hygromycin phosphotransferase gene via double crossover homologous recombination (Supplementary Fig. S2A and B). Using Agrobacterium tumefaciens-mediated transformation (ATMT), we obtained hygromycin-resistant transformants. Among these transformants, we selected bialaphossensitive transformants for exclusion of ectopic insertions and obtained three transformants having the phenotype with hygromycin resistance and bialaphos sensitivity. Successful replacement of the targeted CoWHI2 gene in these transformants was confirmed by Southern blot analysis.

\section{CoWHI2 is not involved in cell-wall integrity.}

In $S$. cerevisiae, Whi2p is a regulator for transcription factor Msn2p, which regulates expression of stress responsive genes in stress conditions (Sadeh et al. 2011). In the M. oryzae mutant, Momsn2d, a homolog of $S$. cerevisiae Msn2p, showed increased tolerance to calcofluor white $(\mathrm{CFW})$ that disturbs fungal cellwall integrity due to binding to polysaccharides (Zhang et al. 2014). Thus, the regulator of Msn 2 might be involved in cell-wall integrity in response to stress conditions. Therefore, to elucidate whether CoWHI2 is involved in cell-wall integrity, we examined the hyphal growth of the Cowhi2s mutant on potato dextrose agar (PDA) amended with different wall-perturbing agents, i.e., CFW, Congo red (CR) or sodium dodecyl sulfate (SDS). Hyphal growth of the Cowhi2s mutant on PDA was slightly slower than that of the wild type. On SDS, hyphal growth of the Cowhi2s mutant was inhibited nearly the same as the wild type, but the Cowhi2s mutant was more tolerant to CFW and CR than the wild type was (Supplementary Fig. S3).

\section{CoWHI2 is not involved in regulating the cellular} localization of CoRas2 during appressorium formation.

In our previous report, we showed the dynamic cellular localization of small GTP-binding protein CoRas2 during appressorium formation in $C$. orbiculare, suggesting that the regulation of the cellular localization of CoRas2 is important for infection-related morphogenesis and pathogenesis (Harata and Kubo 2014). WHI2 is known to contribute to the appropriate localization of Rasp via actin dynamics in response to nutritional sensing in $S$. cerevisiae (Leadsham et al. 2009). Therefore, to elucidate whether CoWHI2 is involved in the regulation of the dynamic cellular localization of CoRas 2 in C. orbiculare, we examined CoRas2 localization in the Cowhi2s mutant during appressorium formation. Cellular localization of CoRas2 in the Cowhi2s mutant was similar to that of the wild type, indicating that CoWHI2 does not contribute to the cellular regulation of CoRas2, differing from the case in S. cerevisiae (Supplementary Fig. S4).

\section{Host defense response with callose deposition is strongly induced by the Cowhi2s mutant in the initial infection stage.}

To elucidate whether CoWHI2 is involved in fungal pathogenesis, detached cucumber cotyledons were inoculated with the Cowhi2s mutant. The frequency of lesions larger than $0.4 \mathrm{~mm}$ in diameter caused by the Cowhi2s mutant was lower than that caused by the wild type and by transformants with CoWHI2 reintroduced. The frequency of lesions smaller than

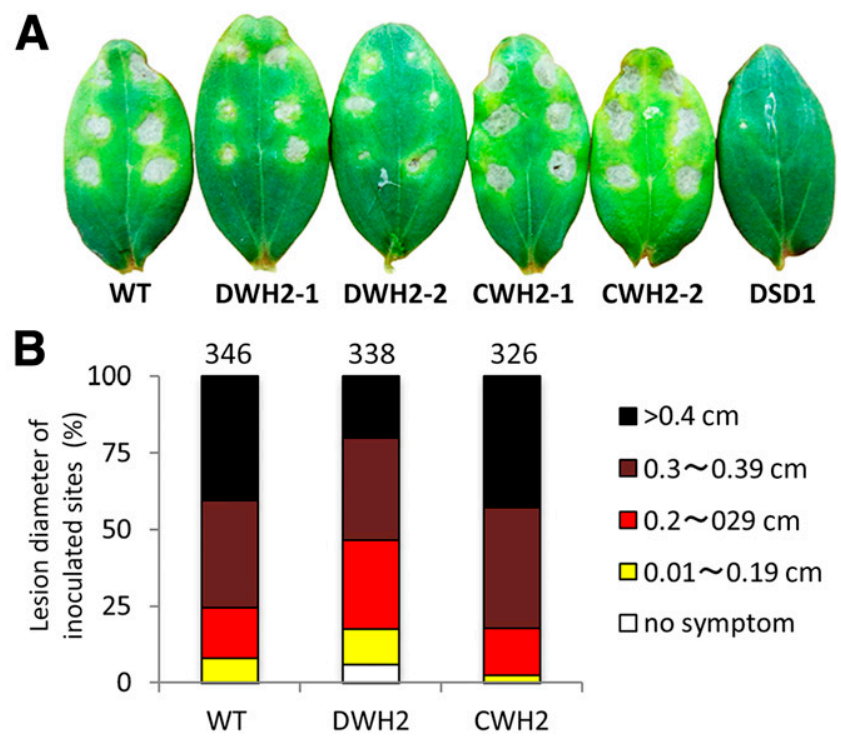

Fig. 1. Pathogenicity assay of Colletotrichum orbiculare whi2s mutants on cucumber cotyledons. A, Conidial suspensions of each strain were placed on detached cotyledons of cucumber and were incubated at $24^{\circ} \mathrm{C}$ for 5 days. WT = wild-type 104-Tn DWH2-1 and DWH2-2 = Cowhi2s mutants CWH2-1 = CoWHI2-complemented transformant of DWH2-1n CWH2-2 = CoWHI2-complemented transformant of DWH2-2n and DSD1 = Cossd1A mutant. B, Virulence of the wild type and the Cowhi2s mutant was scored at 5 days postinoculation. The diameter of the lesion caused by each strain was measured and was scored as shown. The total number of inoculation sites is indicated above each column. 
$0.29 \mathrm{~mm}$ in diameter caused by the Cowhi2d mutant was higher than that caused by the wild type and transformants with CoWHI2 reintroduced, indicating that $C$. orbiculare CoWHI2 is required for full virulence (Fig. 1). In $C$. orbiculare, we reported that the Cossd1 $1 \Delta$ mutant that is defective in the constitution of its cell wall was less virulent to its host, due to an increased host defense response accompanied by rapidly

A
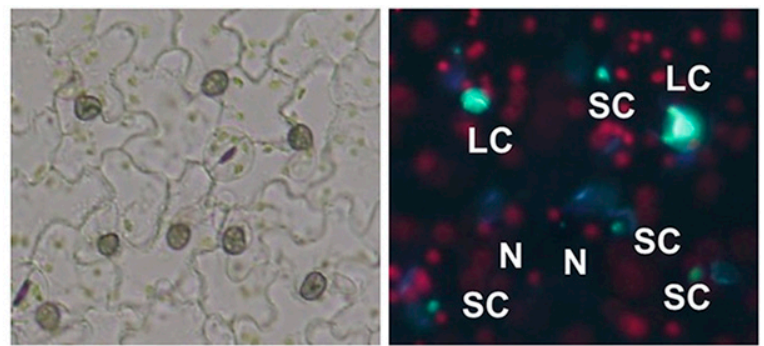

B
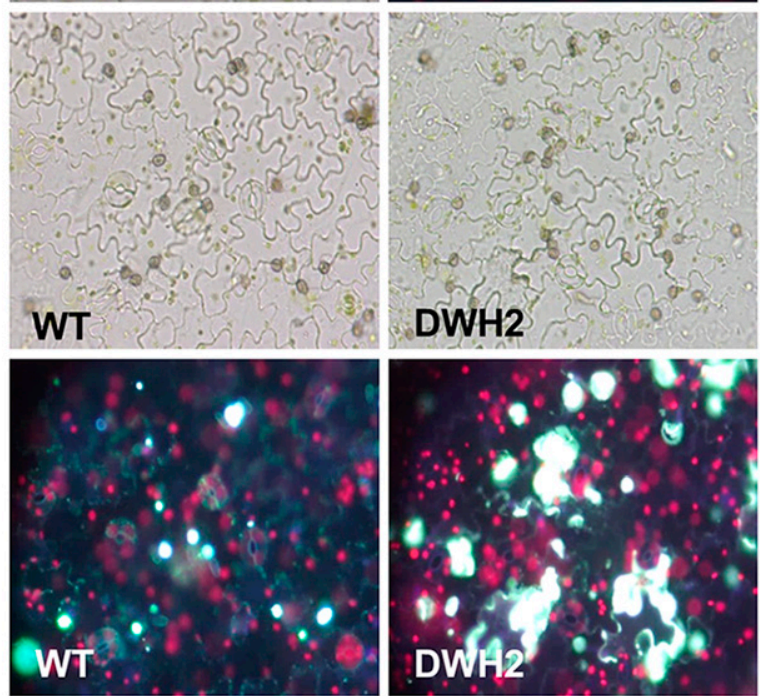

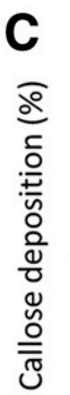

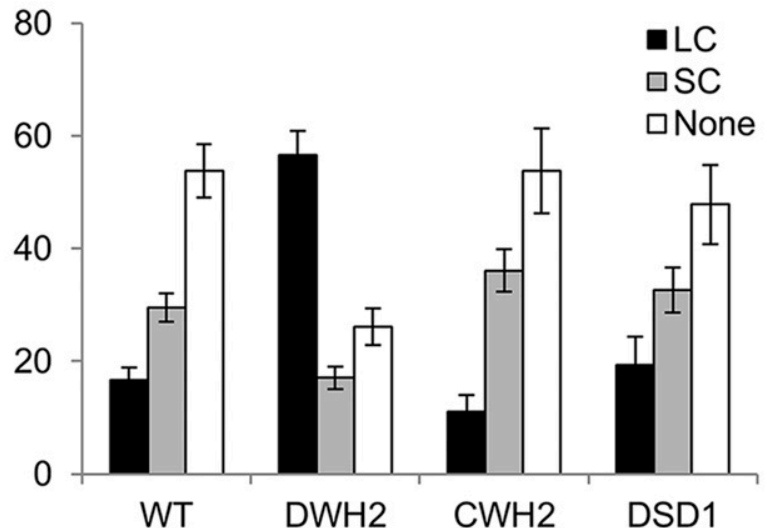

Fig. 2. Callose deposition in epidermal cells on the abaxial surface of cucumber cotyledons beneath appressoria of the Cowhi $2 \Delta$ mutant. Conidial suspensions of the strains were placed on the abaxial surface of cucumber cotyledons; after incubation at $24^{\circ} \mathrm{C}$ for $24 \mathrm{~h}$, cotyledons were stained with aniline blue and were examined for callose deposition, using fluorescence microscopy. A, Variation in size of callose deposition induced by Colletotrichum orbiculare. LC indicates callose deposits larger than the boundary of the appressorium; SC, callose deposits smaller than the boundary of the appressorium; $\mathrm{N}$, none detected. $\mathbf{B}$, Callose deposition beneath appressoria formed by the wild type and the Cowhi2s mutant. C, Mean percentage of the sizes of callose deposition beneath appressoria formed by each strain. Approximately 200 appressoria were examined per inoculation site, three replicates were examined for each of three independent experiments; standard errors are indicated. WT $=$ wildtype 104-Tn DWH2 = Cowhi2s mutantn CWH2 = CoWHI2-complemented transformant of DWH2n and DSD1 $=$ Cossd1 $1 \Delta$ mutant. induced callose deposition at sites where appressoria tried to penetrate (Tanaka et al. 2009).

To elucidate whether this type of host defense response accompanied by callose deposition is induced by attack of the Cowhi2s mutant, we monitored callose deposition on cucumber epidermal cells beneath appressoria of the Cowhi2s mutant at $24 \mathrm{~h}$ postinoculation (hpi). By $24 \mathrm{hpi}$, large deposits of callose had accumulated at high frequency beneath these appressoria compared with the wild type (Fig. 2). Interestingly, the callose induced by the Cowhi2 $\Delta$ mutant accumulated at around $24 \mathrm{hpi}$, while the callose induced by the Cossd1 $1 \Delta$ mutant accumulated after 48 hpi. These data indicated that the deposition
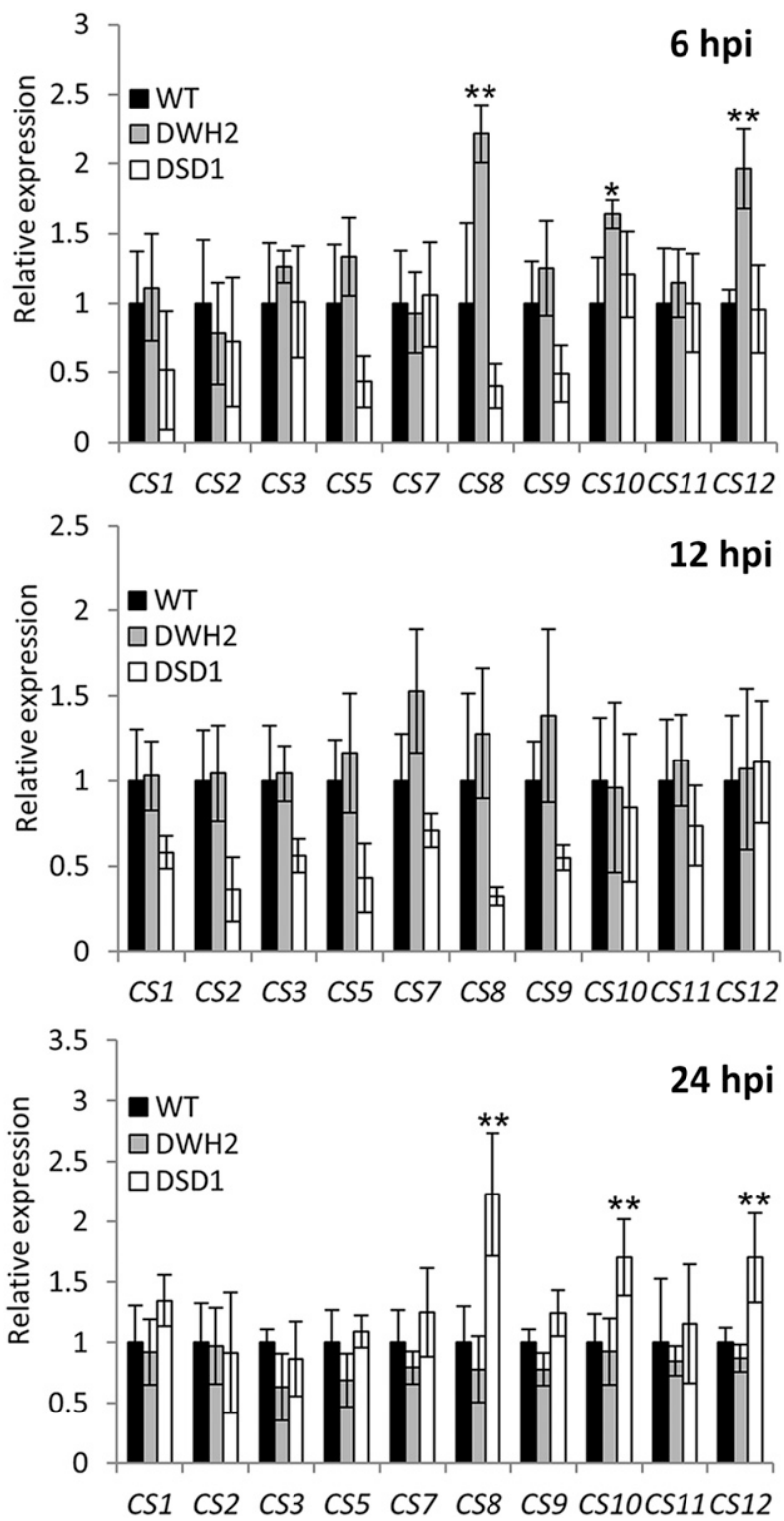

Fig. 3. Expression of callose synthase genes in cucumber cotyledons inoculated with the Cowhi2s mutant. Conidial suspensions of each strain were placed on detached cotyledons of cucumber, which were incubated for 6,12 , or $24 \mathrm{~h}$. Total RNA was extracted from the incubated leaves, and expression of callose synthase genes was analyzed by quantitative reverse transcription-polymerase chain reaction. Three independent experiments were conducted; standard errors are indicated. Asterisks represent significant differences between cucumber leaves inoculated with the wild type and each mutant (Student's $t$ test: one asterisk $(*)$ indicates $P<0.05$ and two (**) $P<0.01$ ). WT $=$ wild-type $104-\mathrm{T}, \mathrm{DWH} 2=$ Cowhi2 $\Delta$ mutant, and DSD $1=\operatorname{Coss} 11 \Delta$ mutant. 
of callose induced by the Cowhi2 $\Delta$ mutant temporally differed from that induced by the Cossd $1 \Delta$ mutant.

To test this hypothesis, we analyzed expression patterns of callose synthase genes of Cucumis sativus that represent homologs of Arabidopsis thaliana GSL genes involved in callose biosynthesis (Richmond 2000; Verma and Hong 2001) (Supplementary Fig. S5). Quantitative reverse transcriptionpolymerase chain reaction (qRT-PCR) analysis showed that, in cucumber inoculated with the Cowhi2 $\Delta$ mutant, the expression of callose synthases 8,10 , and $12(C S 8, C S 10, C S 12)$, at 6 hpi, was 2.2-, 1.6- ,and 1.9-fold higher, respectively, than after inoculation with the wild type (Fig. 3). At 12 hpi, the expression of callose synthase genes was not significantly induced by the Cowhi2s mutant or the Cossd1s mutant compared with those inoculated with the wild type. At $24 \mathrm{hpi}$, the expression of CS8, CS10, and CS12 in cucumber leaves inoculated with the Cossd1s mutant was 2.2-, 1.7-, and 1.7-fold higher, respectively, than in cucumber leaves inoculated with the wild type. Therefore, callose synthase genes $C S 8, C S 10$, and $C S 12$ are rapidly induced in the host cucumber leaves at the initial infection stage of Cowhi2s mutant.

\section{Pleiotropic host defense responses induced by the Cowhi2s mutant.}

$\mathrm{H}_{2} \mathrm{O}_{2}$ production by the host plant after attack by phytopathogenic fungi is one of the key factors for activating host defense responses. To elucidate whether $\mathrm{H}_{2} \mathrm{O}_{2}$ production in epidermal cells is induced by the Cowhi2s mutant, we evaluated $\mathrm{H}_{2} \mathrm{O}_{2}$ accumulation in epidermal cells beneath appressoria of the Cowhi2s mutant by treating with 3,3'-diaminobenzidine (DAB) to detect hydrogen peroxide. The epidermal cells beneath appressoria of the Cowhi2s mutant stained strongly with
DAB compared with the wild type, indicating that the Cowhi2s mutant does induce a host defense response accompanied by $\mathrm{H}_{2} \mathrm{O}_{2}$ accumulation (Fig. 4A and B). Interestingly, the epidermal cells at attempted penetration sites of the Cowhi2s mutant emitted significantly more autofluorescence compared with the wild type (Fig. 4C and D).

Plant NADPH oxidase is accepted as the key regulator of $\mathrm{H}_{2} \mathrm{O}_{2}$ generation associated with the plant defense response against attack by phytopathogenic fungi (Torres et al. 2005; Yoshioka et al. 2003). The Cowhi2s mutant induced $\mathrm{H}_{2} \mathrm{O}_{2}$ accumulation in cucumber epidermal cells. Therefore, to elucidate whether the plant defense response induced by the Cowhis $\Delta$ mutant is triggered by the activity of NADPH oxidases, we examined callose deposition and $\mathrm{H}_{2} \mathrm{O}_{2}$ accumulation on cucumber epidermal cells beneath appressoria of the Cowhi2s mutant treated with $0.5 \mu \mathrm{M}$ diphenyleneiodonium (DPI), an inhibitor of NADPH oxidase activity. The frequency of callose deposition and $\mathrm{H}_{2} \mathrm{O}_{2}$ accumulation in the Cowhi2s mutant treated with DPI was lower than those in the untreated mutant (Supplementary Fig. S6).

The accumulation of phenolic compounds after pathogen attack has been previously reported (Chi et al. 2009; Nicholson and Hammerschmidt 1992), and our data suggested that the defense reaction of the host plant may be affected by infection with the Cowhi2s mutant. Therefore, to analyze whether plant defense gene expression was affected by infection with the mutant, we analyzed levels of the pathogenesis-related protein $1(P R-1)$ gene and defense-related DEFENSIN19 gene of Cucumber sativus, homologs of PRI and PDF1.2, that are induced by salicylic acid and jasmonic acid in A. thaliana, respectively (Penninckx et al. 1998; Tanaka et al. 2007). At $48 \mathrm{hpi}$, the expression of DEFENSIN19 in cucumber leaves
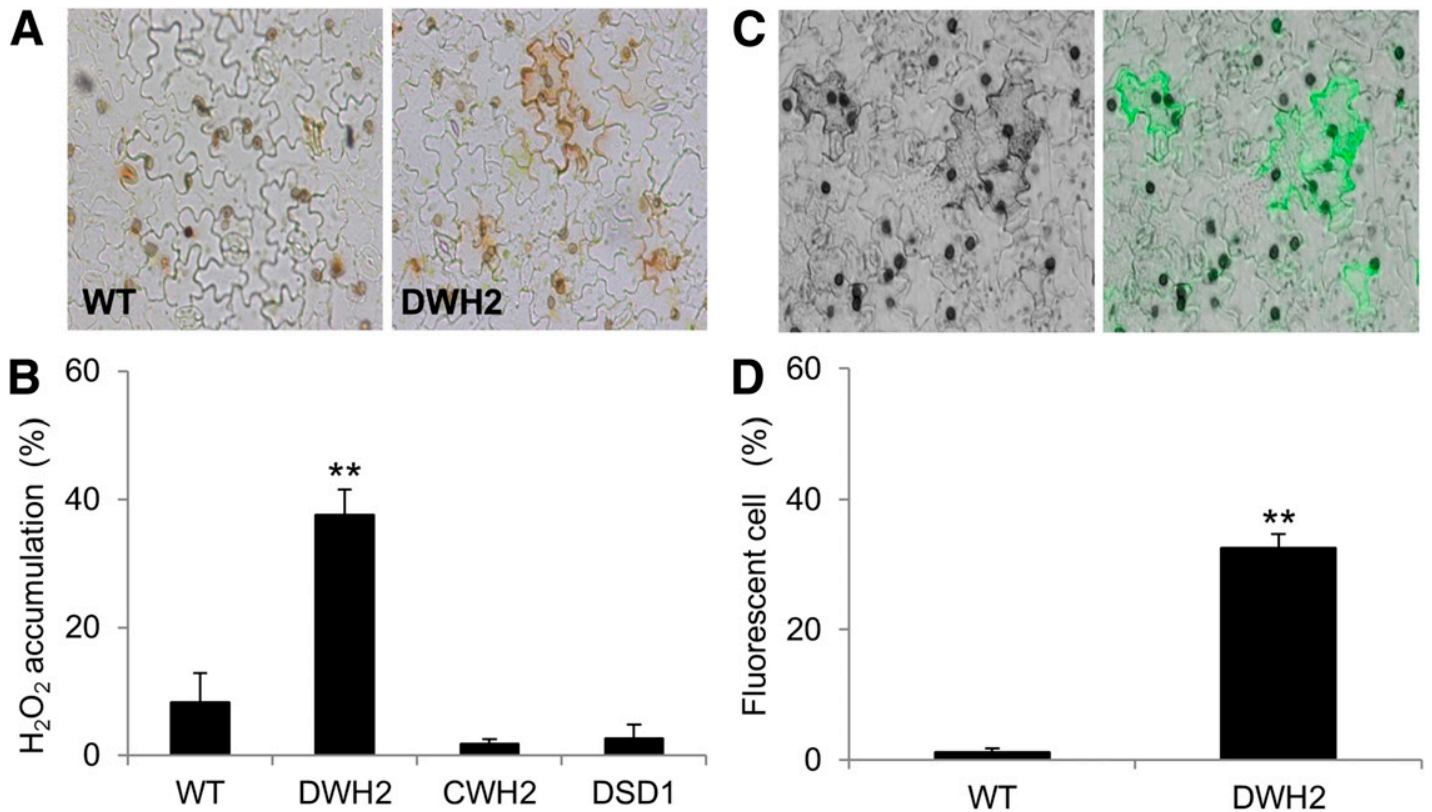

Fig. 4. The Cowhi2 $\Delta$ mutant induced activation of host defense responses. $\mathbf{A}, \mathrm{H}_{2} \mathrm{O}_{2}$ accumulation beneath appressoria of each strain. Conidial suspensions of each strain were placed on the abaxial surface of cucumber cotyledons. After incubation at $24^{\circ} \mathrm{C}$ for $24 \mathrm{~h}$, cotyledons were stained with $3,3^{\prime}$-diaminobenzidine and were then observed with microscopy for dark-brown staining to indicate $\mathrm{H}_{2} \mathrm{O}_{2}$ accumulation. WT = wild-type $104-\mathrm{T}$, DWH2 $=$ Cowhi2 $\Delta$ mutant, $\mathrm{CWH} 2=$ CoWHI2-complemented transformant of DWH2, DSD1 = Cossd1s mutant. B, Mean frequency of $\mathrm{H}_{2} \mathrm{O}_{2}$ accumulation beneath appressoria of each strain. Approximately 100 appressoria of each strain were observed per inoculation site, and three replicates were examined for each of three independent experiments; standard errors are indicated. Asterisks represent significant differences between cucumber leaves inoculated with the wild type and each strain (Student's $t$ test: $P<0.01$ ). C, Fluorescence in cells beneath appressoria formed by the Cowhi2 $\Delta$ mutant. Conidial suspensions of each strain were placed on the abaxial surface of cucumber cotyledons; after incubation at $24^{\circ} \mathrm{C}$ for $48 \mathrm{~h}$, host cells beneath appressoria were examined with fluorescence microscopy. $\mathbf{D}$, Mean frequency of cells with fluorescence beneath appressoria formed by each strain. Approximately 100 appressoria of each strain were observed per inoculated site; three replicates were examined. Three independent experiments were conducted; standard errors are indicated. Asterisks represent significant differences between cucumber leaves inoculated with the wild type and Cowhi2s (Student's $t$ test: $P<0.01$ ).

Vol. 29, No. 6, 2016 / 471 
inoculated with the Cowhi2 $\Delta$ mutant was slightly higher than in the wild type, while the expression of $P R-1$ in cucumber leaves inoculated with the Cowhi2 $\Delta$ mutant was lower than in the wild type (Fig. 5). At $72 \mathrm{hpi}$, the expression of DEFENSIN19 in cucumber leaves inoculated with the Cowhi2s mutant was significantly higher than in the wild type, while the expression of $P R-1$ in cucumber leaves inoculated with the Cowhi2s mutant was lower than in the wild type (Fig. 5). Interestingly, the Cossd1D mutant strongly induced expression of $P R-1$ compared with the wild type. Taken together, these results indicated that the initial host defense responses of host cucumber Cucumber sativus against the attack by the Cowhi2s mutant were much different than in the wild type.

\section{The Cowhi2s mutation affects $C$. orbiculare-Cucumber sativus interactions in the biotrophic phase.}

The Cossd1 $1 \Delta$ mutant is unable to form infection hyphae on the host cells, due to callose deposition at attempted penetration sites below the appressoria, and has attenuated virulence (Tanaka et al. 2009). To determine whether the Cowhi2s mutant can form infection hyphae on cucumber leaves, we observed the infection process of the Cowhi2s mutant. The frequency of infection hyphae in the Cowhi2s mutant was similar to the wild type, and CoWHI2 reintroduced transformants (Fig. 6A and B). Next, to evaluate whether the autofluorescence in epidermal cells induced by the attack of Cowhi2 $\Delta$ mutant was associated with blocked appressorium-mediated penetration by the Cowhi2s mutant, we
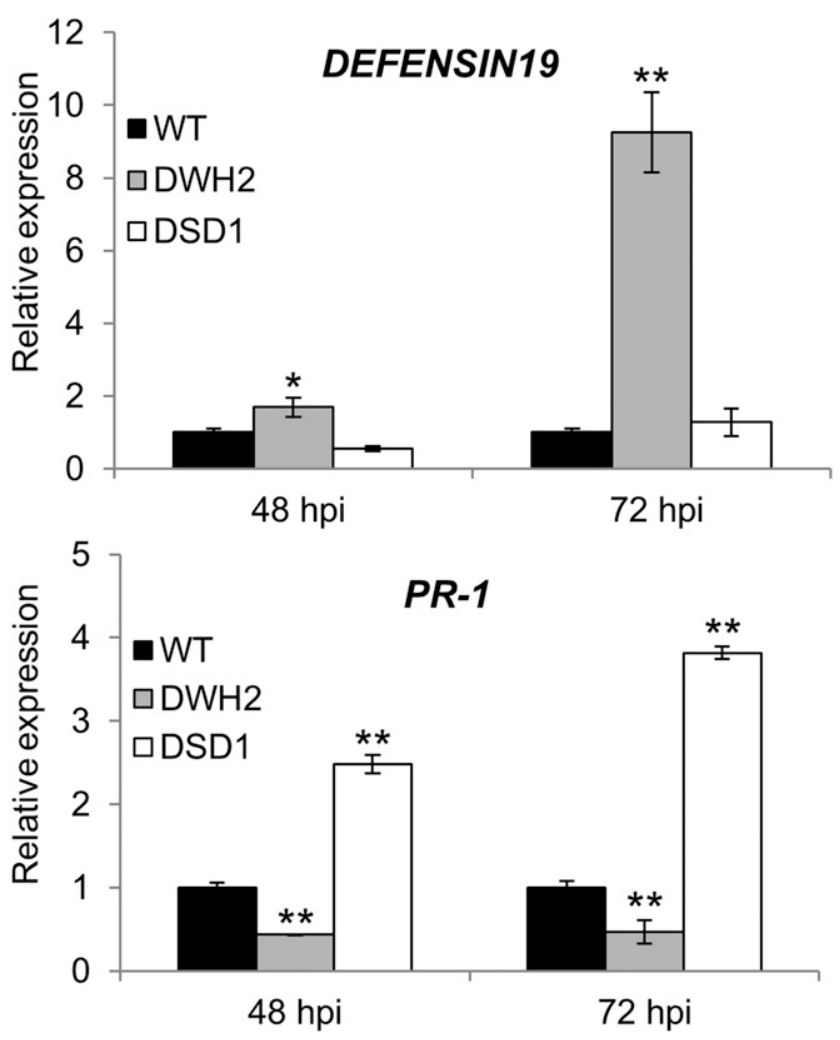

Fig. 5. Expression of DEFENSIN 19 and $P R-1$ in cucumber cotyledons after inoculation with each strain. Conidial suspensions of each strain were placed on the abaxial surface of cucumber cotyledons; at 48 and $72 \mathrm{~h}$ postinoculation (hpi), total RNA was extracted from cotyledons and expression of DEFENSIN19 and $P R-1$ was analyzed by quantitative reverse transcription-polymerase chain reaction. Three independent experiments were conducted and standard errors are indicated. WT = wild-type 104-T; DWH2 = Cowhi $2 \Delta$ mutant; DSD1 $=$ Cossd1 $1 \Delta$ mutant. Asterisks represent significant differences between cucumber leaves inoculated with the wild type and each mutant (Student's $t$ test: one asterisk $(*)=P<0.05$, two $(* *)=$ $P<0.01)$. examined the development of infection hyphae of the mutant at the autofluorescent cells. Interestingly, the autofluorescent epidermal cells were not penetrated and the Cowhi2s mutant did not grow in the host cell (Fig. 6C and D), but nonfluorescent adjacent cells around the autofluorescent cells did not prominently block penetration and infectious growth. These data indicated that the alteration causing or accompanying the autofluorescence in epidermal cells is involved in inhibiting the appressorium-mediated penetration and infectious growth of the Cowhi2s mutant.

Although infection hyphae growth with biotrophy in hemibiotrophic fungi is inhibited under the activation of plant defense responses, infection hyphae growth of the Cowhi2s mutant was not inhibited at 72 hpi when the $C$. orbiculare wild type forms a biotrophic infection hyphae. To elucidate whether the Cowhi2s mutant retains the ability to establish this biotrophic phase, we generated wild type and Cowhi2 $\Delta$ transformants that expressed the fluorescent gene VENUS under the control of a biotrophy-specific promoter and the fluorescent gene $m C h e r r y$ under the control of the constitutively expressed TRANSLATION ELONGATION FACTOR $(T E F)$ promoter derived from Aureobasidium pullulans and, then, assessed biotrophic fluorescence intensity normalized by mCherry signal intensity during the biotrophic stage. First, we selected two biotrophy-specific expression genes, polysaccharide deacetylase family protein $(C o P S D 1)$ and 4-aminobutyrate aminotransferase (CoATF1), that are prominently expressed at higher levels during the biotrophic stage than during appressorium development and the necrotrophic stage, and approximately $2.0 \mathrm{~kb}$ of their $5^{\prime}$ upstream regions and $T E F$ promoter was fused with the VENUS and mCherry genes, respectively (Gan et al. 2013). Then, we successfully generated transformants carrying the biotrophy-specific expression VENUS gene and the constitutively expressed mCherry gene. WT/TEF $\mathrm{Wro}_{\mathrm{p}}$ :mCherry/ $\mathrm{CoPSD}_{\text {pro }}$ :VENUS is the wild type and $\mathrm{DWH}_{2} / \mathrm{TEF}_{\text {pro:mCherry/ }}$ $\mathrm{CoPSD}_{\text {pro }}$ :VENUS is the Cowhi2d mutant transformant, expressing the VENUS gene with the CoPSD1 promoter and the $m$ Cherry gene with the $T E F$ promoter. $\mathrm{WT} / \mathrm{TEF}_{\text {pro: }}: \mathrm{mCherry} / \mathrm{CoATF} \mathrm{F}_{\text {pro }}$ :VENUS is the wild type and DWH2/TEF ${ }_{\text {pro }}:$ mCherry/CoATF $_{\text {pro }}$ :VENUS is the Cowhi2A mutant transformant, expressing the VENUS gene with the CoATF1 promoter and the mCherry gene with the $T E F$ promoter. At $72 \mathrm{hpi}$, the frequency and intensity of the CoPSD $_{\text {pro: }}$ VENUS signal in infection hyphae of Cowhi2s was lower and weaker than in those in the wild type (Fig. 7A, C, and D). Similarly, the frequency and the intensity of the $\mathrm{CoATF}_{\text {pro: }}$ : VENUS signal in infection hyphae of Cowhi2s was lower and weaker than those in the wild type (Fig. 7B to D).

CoWhi2 directly interacts with CoPsr1 and the Copsr1D mutant shows a similar phenotype to the Cowhi2s mutant.

In $S$. cerevisiae, Whi2p directly interacts with the phosphatase Psr1p and the complex dephosphorylates the transcription factor Msn2p. Therefore, to analyze whether CoWhi2 interacts with CoPsr1, the homolog of S. cerevisiae Psr1p, we performed yeast two-hybrid assays. First, based on $C$. orbiculare genomic information, we searched for a homolog of $S$. cerevisiae PSRI in $C$. orbiculare and found gene ENH79657, which putatively encodes a 513-aa protein (Gan et al. 2013). The predicted amino acid sequence encoded by CoPSR 1 is significantly similar to that of Psr1p in S. cerevisiae (Supplementary Fig. S7). Next, we constructed the bait CoWHI2 vector (BD-CoWhi2) and prey CoPSR1 vector (AD-CoPsr1) and these vectors were used to transform the Gold and Y187 yeast strains, respectively. In the yeast two-hybrid assays, the mating type carrying BD-CoWhi2 and AD-CoPsr1 grew on selective medium, while growth of the negative control was inhibited on selective medium, indicating that CoWhi2 directly interacts with CoPsr1 in C. orbiculare as in $S$. cerevisiae Whi2p-Psrlp (Fig. 8A). To elucidate whether the function of CoPSRl is similar to that of CoWHI2 during 
infection by $C$. orbiculare, we conducted a functional analysis of CoPSR1 with the CopsrlA mutant. In a virulence test, the Copsrld mutant caused smaller lesions than the wild type did on cucumber cotyledons (Fig. 8B). Next, to elucidate whether the Copsrld mutant induces a host defense response similar to that induced by the Cowhi2s mutant, we monitored callose deposition on cucumber cotyledons beneath appressoria of the Copsr1s mutant. The frequency of large callose depositions beneath appressoria of the Copsrl $\Delta$ mutant was higher than in the wild type at $24 \mathrm{hpi}$ (Fig. 8C). Taken together, the similarity of the phenotype between the Cowhi2s mutant and the Copsrls mutant supported the possibility that CoWhi2 and CoPsr1 work by forming a functional complex as in S. cerevisiae.

\section{CoWHI2 is involved in TOR-dependent upregulation of ribosomal gene expression.}

The transcription factor Msn2p is known to be dephosphorylated by the Whi2p-Psr1p complex and to bind to the core consensus 5' -WAGGGG-3' of stress response element (STRE) genes and to regulate STRE gene expression in S. cerevisiae (Görner et al. 1998; Martínez-Pastor et al. 1996) Therefore, to explore which genes are regulated by CoWhi2, we comprehensively analyzed gene expression in the Cowhi2s mutant at 4 hpi with a microarray, since expression of $C S 8, C S 10$, and CS12 in the cucumber inoculated with the Cowhi2s mutant significantly increased at $6 \mathrm{hpi}$. This analysis revealed that 58 genes were upregulated and 21 genes were downregulated (>10-fold change) in the Cowhi2s mutant compared with the wild type (Table 1; Supplementary Table S1). Interestingly, 44 genes among the upregulated genes ( $>10$-fold change) in the Cowhi2s mutant were ribosomal protein-related genes (Table 1).

Ribosome biogenesis in response to environmental conditions is crucial in regulating cell growth and cellular metabolism
(Martin et al. 2004). In mammals and S. cerevisiae, TOR is known to be a regulatory factor of ribosomal gene expression in response to environmental conditions (Crespo and Hall 2002; Martin et al. 2006). Therefore, we hypothesized that expression of ribosomal genes in the Cowhi2s mutant are upregulated by the activation of TOR. To verify this hypothesis, we performed a microarray focusing on ribosomal gene expression of the Cowhi2 $\Delta$ mutant at 4 hpi treated with $100 \mathrm{nM}$ rapamycin under conditions that decrease TOR activity. Surprisingly, ribosomal gene expression in the Cowhi2s mutant treated with rapamycin was significantly downregulated compared with the untreated mutant (Fig. 9). In M. oryzae, expression of MoRS 2 and MoRS3 genes encoding ribosomal proteins is upregulated when TOR is activated but downregulated when TOR is inactivated (Fernandez et al. 2014; Marroquin-Guzman and Wilson 2015). We analyzed expression of CoRS2 and CoRS3 in the Cowhi2s mutant, homologs of MoRS2 and MoRS3, by qRT-PCR with or without $100 \mathrm{nM}$ rapamycin, at $4 \mathrm{hpi}$ (Supplementary Fig. S8). The expression of CoRS2 and CoRS3 in the Cowhi2s mutant was upregulated compared with the wild type, while expression of CoRS2 and CoRS3 in the Cowhi2s mutant treated with rapamycin was downregulated compared with the untreated mutant (Fig. 10A and B). Taken together, these data suggested that expression of ribosomal genes in the Cowhi2s mutant is upregulated by activation of TOR.

Because the activation of the TOR pathway promotes biosynthesis of ribosomal proteins and translational initiation, resulting in the control of cell growth and cellular metabolism, we considered that the phenotype of the Cowhi2s mutant and the Copsrls mutant was caused by a disturbance of the wildtype hemibiotrophic parasitic mode through the activation of TOR. To examine this hypothesis, we monitored callose
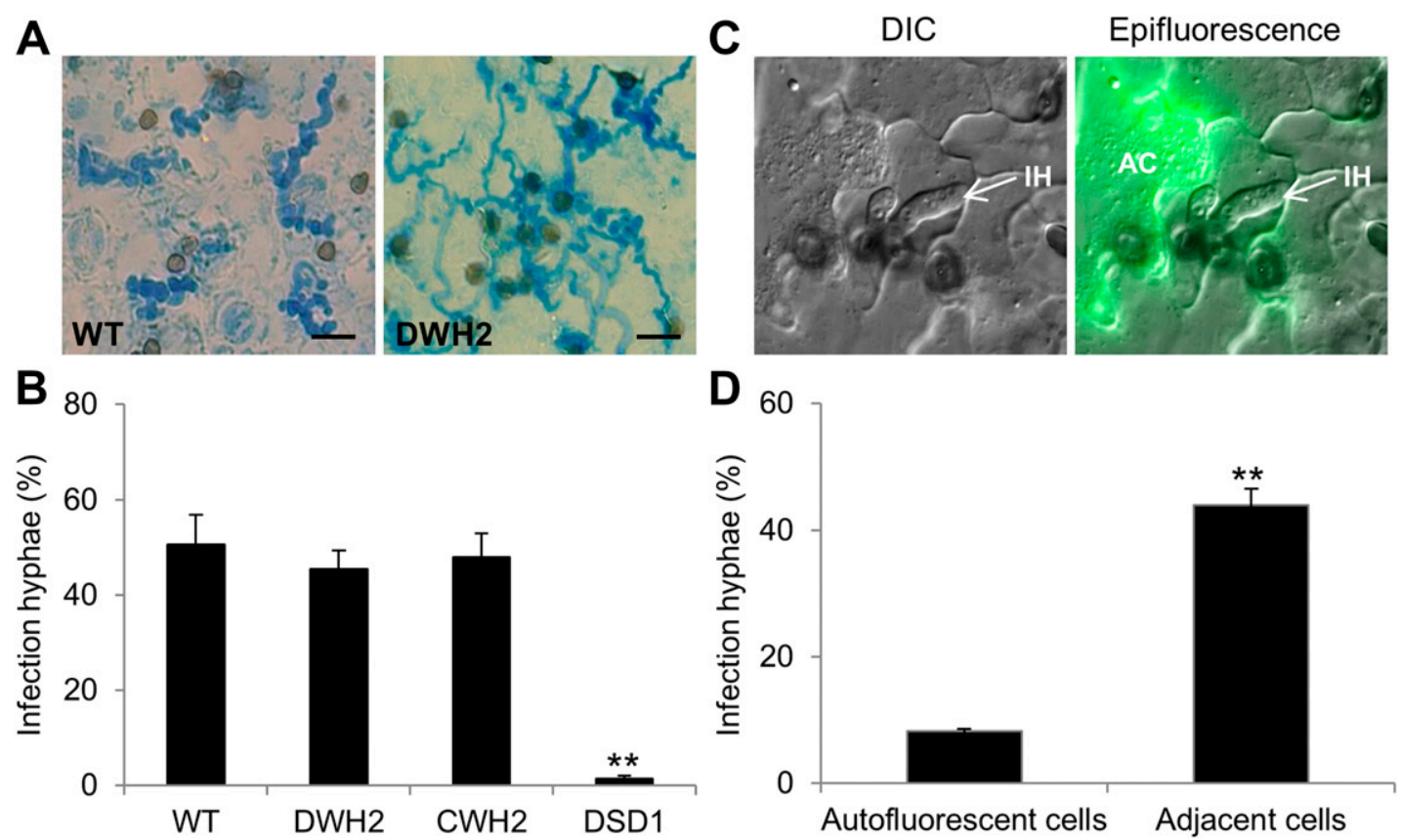

Fig. 6. Infection hyphae formed by the Cowhi $2 \Delta$ mutant on cucumber cotyledons. A, Infection hyphae development of wild type (WT) and Cowhi $2 \Delta$ mutant at $72 \mathrm{~h}$ postinoculation. Cotyledons were inoculated with a conidial suspension of the strain and were incubated at $24^{\circ} \mathrm{C}$. WT $=$ wild-type $104-\mathrm{T}$ and $\mathrm{DWH} 2=\mathrm{Cowhi} 2 \Delta$ mutant. Scale bar, $20 \mu \mathrm{m}$. B, Frequency of infection hyphae of the Cowhi2 mutant in epidermal cells of the abaxial surface of cotyledons. Approximately 100 appressoria of each strain were observed per inoculated site. Three independent experiments were conducted and standard errors are indicated. WT $=$ wild-type 104-T, DWH2-1 = Cowhi2s mutant, CWH2 = CoWHI2-complemented transformant of DWH2, and DSD1 = Cossd1 $\Delta$ mutant. Asterisks represent significant differences between the wild type and each mutant (Student's $t$ test: $P<0.01$ ). C, Infection hyphae formed by the Cowhi2 $\Delta$ mutant in adjacent cells of autofluorescent cells. IH = infection hyphae and $\mathrm{AC}=$ autofluorescent cell. D, Frequency of infection hyphae formed by the Cowhi2 $\Delta$ mutant in autofluorescent cells and the adjacent cells beneath approximately 100 appressoria of each strain. Three replicates were examined for each strain in three independent experiments; standard errors are indicated. Asterisks represent significant differences between the wild type and Cowhi2 $\Delta$ mutant (Student's $t$ test: $P<0.01)$. 
deposition in cucumber epidermal cells beneath appressoria formed by the Cowhi2 $\Delta$ mutant and the Copsr $1 \Delta$ mutant treated with $100 \mathrm{nM}$ rapamycin. The frequency of callose deposition beneath appressoria formed by the wild type treated with rapamycin was similar to the wild type without rapamycin treatment, while the frequency of the large callose deposits beneath appressoria of the Cowhi2s mutant and the Copsrls mutant with rapamycin was lower than in the untreated mutant (Fig. 10C to E). Moreover, when we examined the biotrophic stage of the Cowhi2s mutant treated with $100 \mathrm{nM}$ rapamycin, the intensity of the CoPSD $1_{\text {pro }}$ :VENUS signal and

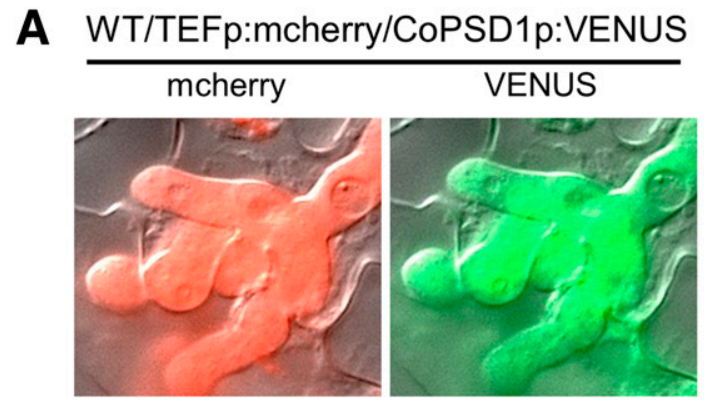

B WT/TEFp:mcherry/CoATF1p:VENUS

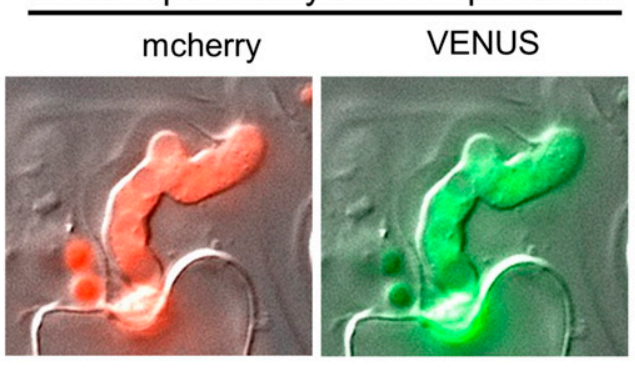

the CoATF $1_{\text {pro: }}$ VENUS signal in the Cowhi $2 \Delta$ mutant treated with rapamycin were stronger than in those without rapamycin treatment (Fig. 10F). These data indicated that CoWHI2 and CoPSR I controls hemibiotrophic infection through TOR during infection of host epidermal cells.

In the $S$. cerevisiae cell cycle, G1 arrest is essential to change from a growth phase to a quiescence phase in response to nutrient starvation and WHI2 is required for this G1 arrest (Radcliffe et al. 1997). In $S$. cerevisiae, TOR inactivation by rapamycin treatment or TORI and TOR2 double deletion can arrest G1 progression in the cell cycle (Loewith and Hall 2011). On the basis of the data

\section{DWH2/TEFp:mcherry/CoPSD1p:VENUS}

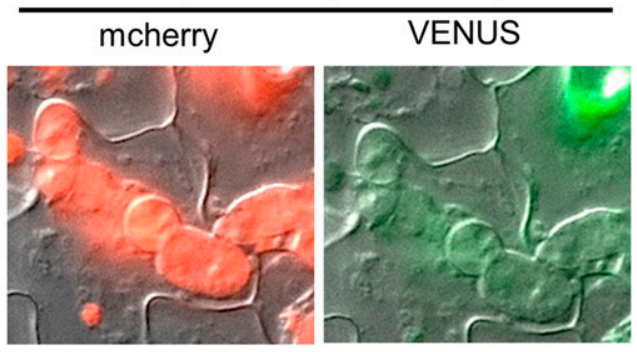

DWH2/TEFp:mcherry/CoATF1p:VENUS

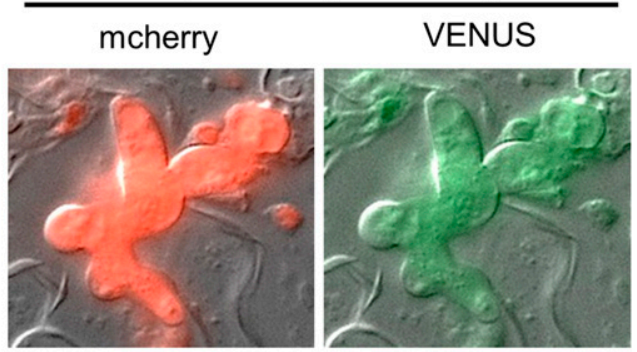

C

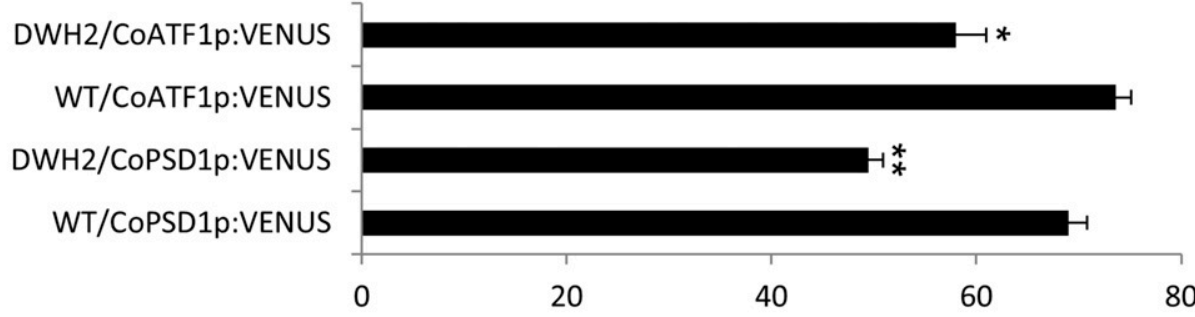

VENUS signal in infection hyphae (\%)

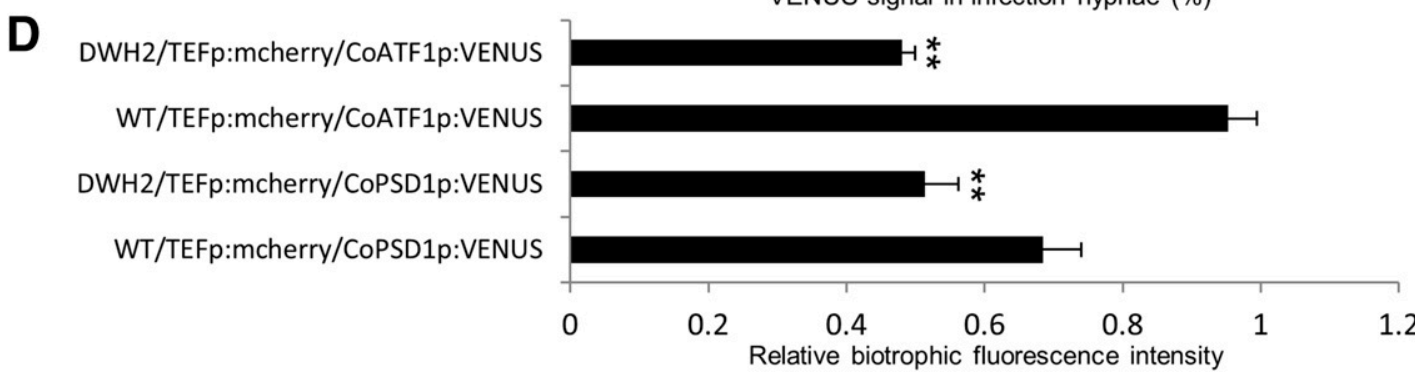

Fig. 7. Fluorescence visualization of expression of biotrophic-specific expression genes in the infection hyphae of the Cowhi2s mutant on cucumber cotyledons at $72 \mathrm{~h}$ postinoculation. A, Biotrophic-specific fluorescent signal detection in the infection hyphae of the $C$. orbiculare whi2 $\Delta$ mutant carrying $T E F_{\text {pro: }}: m$ Cherry and CoPSD1 pro:VENUS or $\mathbf{B}, T E F_{p r o}: m C h e r r y$ and $C o A T F 1_{p r o}: V E N U S$ in cucumber cotyledons. Conidial suspensions of each strain that carried the fluorescence gene with the biotrophic-specific expression promoter were placed on the abaxial surface of cucumber cotyledons and were incubated at $24^{\circ} \mathrm{C}$ for $72 \mathrm{~h}$. VENUS and mCherry fluorescence signals were observed using fluorescence microscopy. $\mathbf{C}$, Frequency of the VENUS signal in the infection hyphae of the Cowhi $2 \Delta$ mutant. Approximately 50 infection hyphae were observed per inoculated site and three replicates were examined. Three independent experiments were conducted; standard errors are indicated. Asterisks represent significant differences between cucumber leaves inoculated with the wild type and Cowhi2 $\Delta$ mutant (Student's $t$ test: two asterisks $(* *)=P<0.01$, one $(*)=P<0.05$ ). D, Quantification of fluorescence intensity of biotrophy-specific

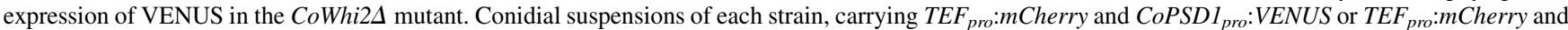
$C O A T F 1_{\text {pro: }}$ VENUS, were placed on the abaxial surface of cucumber cotyledons and were incubated for $72 \mathrm{~h}$. At least 50 VENUS fluorescence signals from infection hyphae were quantified for each strain. The mean biotrophic fluorescence intensity value was calculated by dividing the VENUS fluorescence intensity by the mCherry fluorescence intensity in infection hyphae of each strain. Asterisks represent significant differences between the wild type and Cowhi2s mutant (Student's $t$ test: $P<0.01$ ). 
from these reports, suppression of the Cowhi2 $\Delta$ mutant phenotype by rapamycin treatment could be due to control of G1 progression accelerated by CoWHI2 deletion through TOR inactivation by rapamycin treatment. Therefore, to elucidate whether CoWHI2 is involved in cell-cycle regulation, we generated a Cowhi2s mutant transformant expressing the histone $\mathrm{H} 1$-green fluorescent protein (GFP) fusion protein, which mainly localizes to the nucleus and, then, examined the cells for histone H1-GFP fluorescence signals (Fukada and Kubo 2015). During appressorium formation, the time course of nuclear division of the Cowhi2s mutant did not differ significantly from that of the wild type, while in the Cobub2s mutant, G1/S progression was accelerated during appressorium formation (Fig. 11).

\section{DISCUSSION}

\section{C. orbiculare regulates the biotrophic stage of hemibiotrophic infection via CoWhi2.}

It is generally accepted that plant-pathogenic fungi transmit a variety of extracellular signals such as physical signals and plant signals via an intracellular signaling pathway, resulting in the induction of infection-related morphogenesis and metabolism

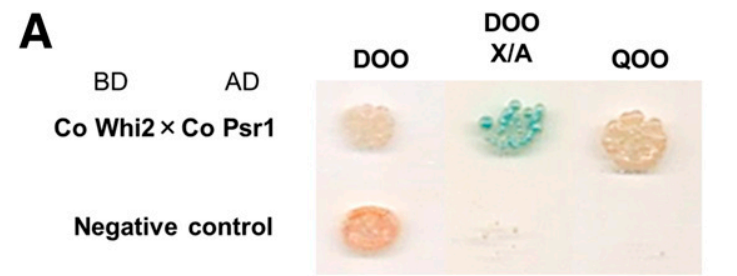

B
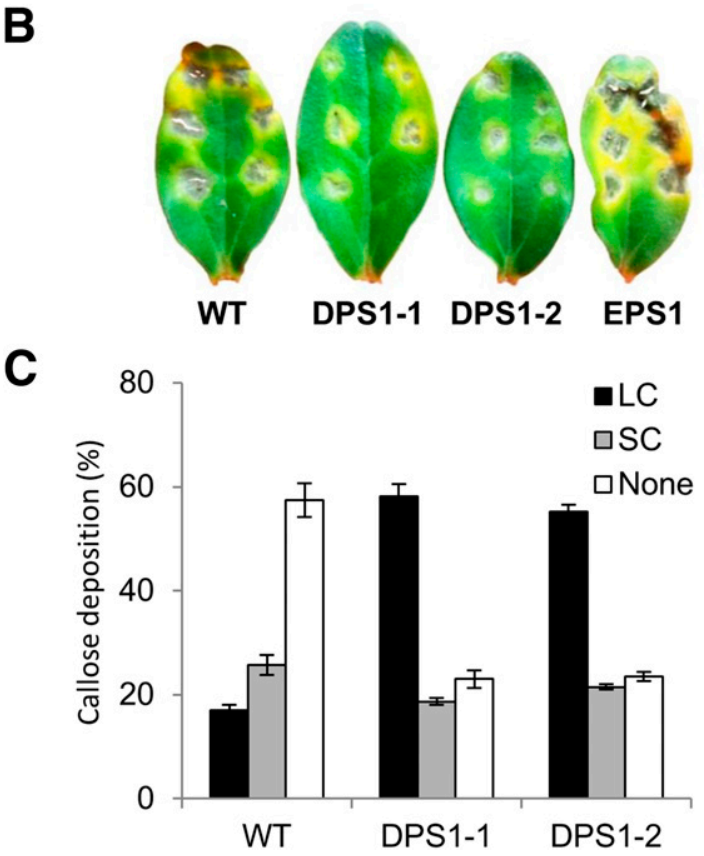

Fig. 8. CoPsr1 directly interacted with CoWhi2, and the phenotype of the Copsrls mutant was similar to that of the Cowhi $2 \Delta$ mutant. A, Colony growth of each strain incubated at $28^{\circ} \mathrm{C}$ for 4 days on the selective medium. Negative control: pGBKT-Lam $\times$ pGADT7-T. B, Conidial suspensions of each strain were placed on detached cotyledons of cucumber and, then, were incubated at $24^{\circ} \mathrm{C}$ for 7 days. Shown are leaves inoculated with wildtype 104-T (WT), DPS2-1 and DPS2-2, the Copsr1D mutant, and EPS1, the ectopic strain. C, Mean frequency of each callose deposition type beneath appressoria of each strain at $24 \mathrm{~h}$ postinoculation. Approximately 200 appressoria of each strain were observed per incubated site; three replicates were examined per strain in each of three independent experiments. Standard errors are indicated.
(Kubo and Takano 2013; Wilson and Talbot 2009). In Ustilago maydis, plant surface-sensing factors Sho1 and Msb2 that act upstream of MAPK Kpp2 and Kpp6 are involved in appressorium formation (Lanver et al. 2010) In M. oryzae, Sho1 recognizes rice leaf wax as a signal for appressorium formation, while Msb2

Table 1. Upregulated genes (10-fold change) in the Cowhi2s mutant compared with the wild type (WT) at $4 \mathrm{~h}$ postinoculation

\begin{tabular}{|c|c|c|}
\hline Target ID & Description & $\begin{array}{l}\text { Whi2 } 2 \text { /WT } \\
\text { fold change }\end{array}$ \\
\hline Cob_07269 & $\begin{array}{l}\text { NAD-dependent epimerase/dehydratase } \\
\text { family protein }\end{array}$ & 26.41 \\
\hline Cob_03272 & $60 \mathrm{~S}$ acidic ribosomal protein $\mathrm{P} 0$ & 18.51 \\
\hline Cob_00779 & 40S ribosomal protein $S 26$ & 18.37 \\
\hline Cob_10175 & $40 \mathrm{~S}$ ribosomal protein $\mathrm{S} 18$ & 18.36 \\
\hline Cob_12426 & 60 S ribosomal protein L28 & 17.37 \\
\hline Cob_02165 & $60 \mathrm{~S}$ acidic ribosomal protein & 16.89 \\
\hline Cob_00778 & Ubiquitin & 16.55 \\
\hline Cob_12837 & $60 \mathrm{~S}$ ribosomal protein $\mathrm{L} 37 \mathrm{a}$ & 16.27 \\
\hline Cob_13019 & 60 S ribosomal protein L24 & 16.22 \\
\hline Cob_09495 & $60 \mathrm{~S}$ ribosomal protein $\mathrm{L} 22$ & 16.17 \\
\hline Cob_07358 & $60 \mathrm{~S}$ ribosomal protein $\mathrm{L} 15$ & 15.90 \\
\hline Cob_13262 & hsp70 chaperone & 15.55 \\
\hline Cob_05002 & $40 \mathrm{~S}$ ribosomal protein $\mathrm{S} 4$ & 15.52 \\
\hline Cob_07318 & $40 \mathrm{~S}$ ribosomal protein $\mathrm{S} 23$ & 14.98 \\
\hline Cob_04116 & $60 \mathrm{~S}$ ribosomal protein L3 & 14.22 \\
\hline Cob_00457 & Alternative oxidase & 14.00 \\
\hline Cob_03396 & $60 \mathrm{~S}$ ribosomal protein L6 & 13.93 \\
\hline Cob_05469 & $\begin{array}{l}\text { UDP-glucose dehydrogenase } \\
\text { UDP-ManNAc }\end{array}$ & 13.91 \\
\hline Cob_10298 & $60 S$ ribosomal protein L17 & 13.88 \\
\hline Cob_13037 & Ribosomal protein L14 & 13.84 \\
\hline Cob_03403 & $60 \mathrm{~S}$ ribosomal protein L35 & 13.82 \\
\hline Cob_01703 & 60S ribosomal protein L9 & 12.95 \\
\hline Cob_10141 & $60 \mathrm{~S}$ ribosomal protein $\mathrm{L10a}$ & 12.95 \\
\hline Cob_08099 & $60 \mathrm{~S}$ acidic ribosomal protein $\mathrm{P} 2$ & 12.88 \\
\hline Cob_08240 & 40S ribosomal protein S17 & 12.83 \\
\hline Cob_11718 & $40 \mathrm{~S}$ ribosomal protein $\mathrm{S} 5$ & 12.56 \\
\hline Cob_01807 & $40 \mathrm{~S}$ ribosomal protein $\mathrm{S} 20$ & 12.45 \\
\hline Cob_02278 & Amino acid transporter & 12.43 \\
\hline Cob_11700 & $40 \mathrm{~S}$ ribosomal protein $\mathrm{S} 6$ & 12.43 \\
\hline Cob_02253 & Hypothetical protein & 12.33 \\
\hline Cob_04000 & $40 \mathrm{~S}$ ribosomal protein $\mathrm{S} 10 \mathrm{~b}$ & 12.25 \\
\hline Cob_11705 & 40S ribosomal protein S8-b & 11.91 \\
\hline Cob_12849 & 40S ribosomal protein S12 & 11.82 \\
\hline Cob_03549 & $60 \mathrm{~S}$ ribosomal protein L11 & 11.78 \\
\hline Cob_02224 & $\begin{array}{l}\text { Fumarylacetoacetate hydrolase } \\
\text { family protein }\end{array}$ & 11.73 \\
\hline Cob_05690 & $60 \mathrm{~S}$ ribosomal protein L8 & 11.70 \\
\hline Cob_07988 & G-protein beta subunit & 11.66 \\
\hline Cob_13407 & HSCARG protein & 11.64 \\
\hline Cob_03452 & $60 \mathrm{~S}$ ribosomal protein $\mathrm{L} 33$ & 11.59 \\
\hline Cob_11185 & $60 S$ ribosomal protein L20 & 11.52 \\
\hline Cob_07152 & $60 \mathrm{~S}$ ribosomal protein $\mathrm{L} 23$ & 11.36 \\
\hline Cob_10127 & $60 S$ ribosomal protein L39 & 11.21 \\
\hline Cob_12054 & $60 \mathrm{~S}$ ribosomal protein $\mathrm{L} 7$ & 11.17 \\
\hline Cob_05317 & $60 S$ ribosomal protein L34 & 10.97 \\
\hline Cob_03476 & Ribosomal protein S28e & 10.97 \\
\hline Cob_08116 & $40 \mathrm{~S}$ ribosomal protein $\mathrm{S} 15$ & 10.95 \\
\hline Cob_13450 & Partial/pseudo & 10.82 \\
\hline Cob_10080 & $60 \mathrm{~S}$ ribosomal protein $\mathrm{L} 2$ & 10.79 \\
\hline Cob_05721 & 40S ribosomal protein $\mathrm{S} 27$ & 10.68 \\
\hline Cob_06504 & $\begin{array}{l}\text { Translationally controlled } \\
\text { tumor protein }\end{array}$ & 10.61 \\
\hline Cob_09771 & $60 \mathrm{~S}$ ribosomal protein $\mathrm{L} 44$ & 10.41 \\
\hline Cob_01044 & Hypothetical protein & 10.40 \\
\hline Cob_10929 & $60 S$ ribosomal protein L31 & 10.28 \\
\hline Cob_13099 & $60 S$ ribosomal protein L21-a & 10.27 \\
\hline Cob_06269 & GMP synthase & 10.23 \\
\hline Cob_08573 & Ribosomal protein S5 & 10.21 \\
\hline Cob_13036 & $\begin{array}{l}\text { Nascent polypeptide-associated } \\
\text { complex subunit alpha }\end{array}$ & 10.18 \\
\hline Cob_13030 & 60S ribosomal protein L37 & 10.02 \\
\hline
\end{tabular}




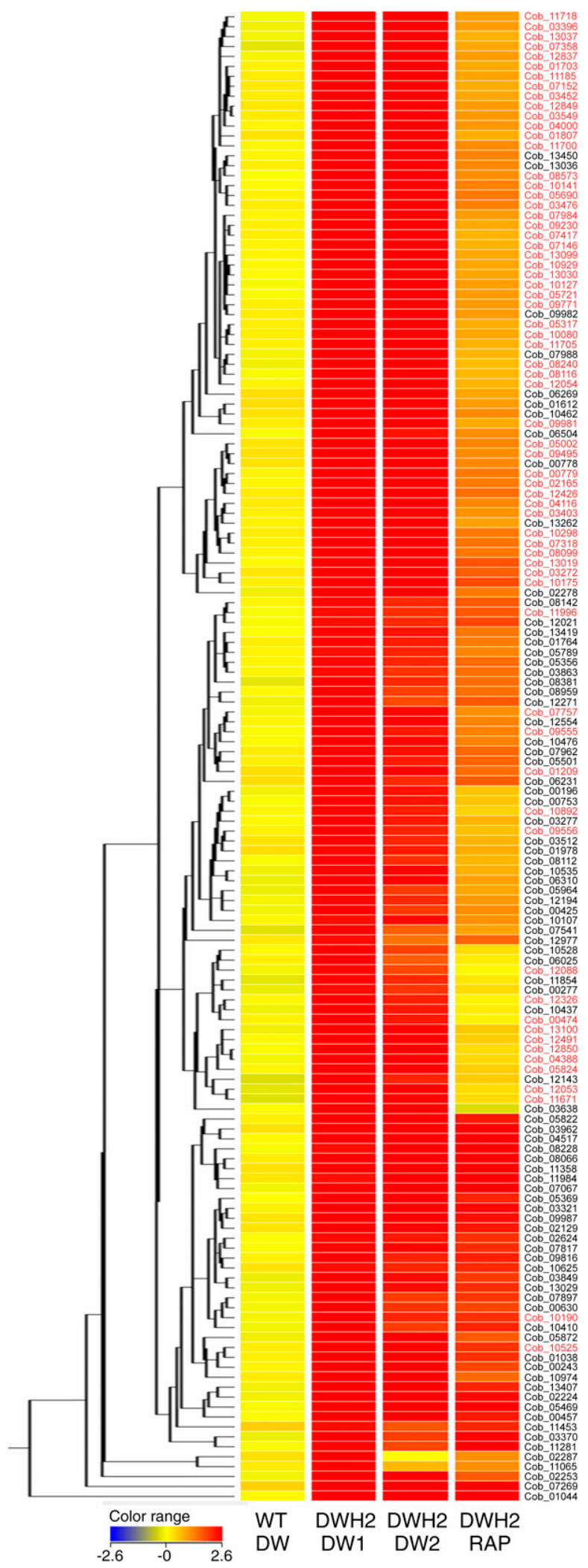

Fig. 9. The upregulated ribosomal gene expression in the Cowhi2 $\Delta$ mutant is regulated by TOR activity. Expression of ribosomal protein-related genes in the Cowhi2s mutant treated with rapamycin or without rapamycin. The heat map of genes depicts the top 150 most highly expressed genes in the Cowhi2s mutant compared with the wild type (WT) at $4 \mathrm{~h}$ postinoculation. WT DW, the WT without rapamycin treatment; DWH2 DW1 and DW2 = the Cowhi2s mutant without rapamycin, DWH2 RAP = Cowhi $2 \Delta$ mutant treated with $100 \mathrm{nM}$ rapamycin. Red text is ribosomal protein-related gene. recognizes surface hydrophobicity and cutin monomers as a signal for appressorium formation (Liu et al. 2011).

As mentioned earlier, hemibiotrophic fungi have two distinctive nutritional stages involved in infection; they initially establish primary bulbous-shaped infection hyphae inside the living host cell (biotrophy) and, subsequently, develop secondary infection hyphae that are narrower and grow rapidly, eventually leading to necrotic lesions at the infected site (necrotrophy). Transcriptome analyses of hemibiotrophic fungi such as $C$. orbiculare and C. higginsianum have revealed that gene expression significantly shifts during the switch from biotrophy to necrotrophy (Gan et al. 2013; O'Connell et al. 2012), but knowledge about the intracellular signaling pathway involved in this hemibiotrophic regulation has been lacking (Wilson and Talbot 2009).

In this study, we first found marked plant responses, rapid callose deposition and $\mathrm{H}_{2} \mathrm{O}_{2}$ production, induced at the initial stage of infection by the Cowhi2s mutant, similar to the earliest host-defense response against necrotrophic fungi such as Botrytis cinerea and Sclerotinia sclerotiorum (Rossi et al. 2011; Williams et al. 2011). In addition, we showed that, at $72 \mathrm{hpi}$, the expression of DEFENSIN19 in cucumber cotyledons inoculated with the Cowhi2s mutant was rapidly induced while the expression of $P R-1$ was lower than in the wild type. A. thaliana $P D F 1.2$, a homolog of DEFENISN19, is also induced after attack by necrotrophic fungi such as $B$. cinerea and Alternaria brassicicola (Rossi et al. 2011; Su'udi et al. 2011). Moreover, the defense response of $A$. thaliana against Alternaria brassicicola activates jasmonic acid-dependent PDF1.2 but suppresses salicylic acid-dependent $P R-1$ (Spoel et al. 2007). Therefore, we considered that the host plant response triggered by the Cowhi2s mutant was similar to that induced by necrotrophic fungi. It is generally considered that the plant defense response against biotrophic pathogens is mainly mediated by salicylic acid-dependent signaling pathways (Glazebrook 2005). Based on data obtained in the present study, $C$. orbiculare first establishes a biotrophic stage in cucumber cotyledons between 48 and 72 hpi. Therefore, it is conceivable that the host defense response, from 48 to $72 \mathrm{hpi}$, against infection by wild-type C. orbiculare is a biotrophic-related plant defense response, in contrast to a necrotrophic infection-related host defense response against infection of the Cowhi2s mutant from 48 to 72 hpi. Therefore, the necrotrophic stage of the Cowhi $2 \Delta$ mutant might occur earlier than the necrotrophic stage in the wildtype $C$. orbiculare.

Moreover, microscopic observations at 72 hpi revealed that the biotrophy-specific fluorescent signal in infection hyphae of the Cowhi2S mutant carrying the VENUS gene with the promoter of a biotrophic-specific gene was less frequent and weaker than in those of the wild type carrying the VENUS gene with the promoter of the biotrophic-specific gene (Fig. 7). Therefore, we assumed that Cowhi2s is a key factor in the regulation of the biotrophic stage in the C. orbiculare-Cucumber sativus interaction.

CoWhi2 regulates the TOR signal pathway and has crucial roles in the biotrophic stage of hemibiotrophic infection in $C$. orbiculare.

The biotrophic, bulbous-shaped infection hyphae in $C$. orbiculare develop in multiple host cells and, subsequently, switch to necrotrophy, but the mechanism underlying this switch in C. orbiculare has been unclear (Kubo and Takano 2013). In the hemibiotrophic fungus $M$. oryzae, TOR signaling is involved in several biological steps in the infection process (Fernandez et al. 2014; Marroquin-Guzman and Wilson 2015). For example, TOR signaling is involved in a checkpoint that controls primary hyphal growth in response to ATP produced via MoTKL1 encoding transketolase and regulates appressorium formation 
by blocking the cAMP-PKA signaling pathway (Fernandez et al. 2014; Marroquin-Guzman and Wilson 2015). Thus, these reports indicate that the role of TOR signaling seems to be involved mainly in processes such as the formation of the appressorium and primary hyphae in hemibiotrophic fungi. In $S$. cerevisiae, Msn2 activity is regulated by Whi 2 and the TOR pathway, but the role of Whi2 in the TOR signaling pathway remains unclear (Sadeh et al. 2011). Although TOR activity regulates the bZIP protein $\mathrm{MeaB}$ and global nitrogen regulator AreA in response to environmental signals through Gln and $\mathrm{NH}_{4}$ and controls virulence functions in Fusarium graminearum (López-Berges et al. 2010), the factors that are involved in transmitting environmental signals to the TOR signaling pathway in hemibiotrophic fungi are far less understood. Based on our analysis of plant responses against the Cowhi2s mutant and the biotrophic stage in the Cowhi2s mutant treated with rapamycin, CoWHI2 seems to be involved in regulating the biotrophic stage of hemibiotrophic infection through the TOR signaling pathway. Hemibiotrophic fungi undergo dynamic variations in gene expression in accordance with the individual infection process, such as appressorium formation, biotrophic infection growth and necrotrophic infection growth (Gan et al. 2013; O'Connell et al. 2012). Therefore, the regulatory system to control the expression of various genes is important for the highly regulated hemibiotrophic infection. In our study, microarray data revealed that many TOR-regulated ribosomal protein genes in the Cowhi2s mutant were notably upregulated compared with the wild type. We speculated that CoWhi 2 has a central role through TOR in the regulation of the biotrophic stage of hemibiotrophic infection. In Magnaporthe oryzae, TOR activation inhibits appressorium formation (Marroquin-Guzman and Wilson 2015). In this study, we
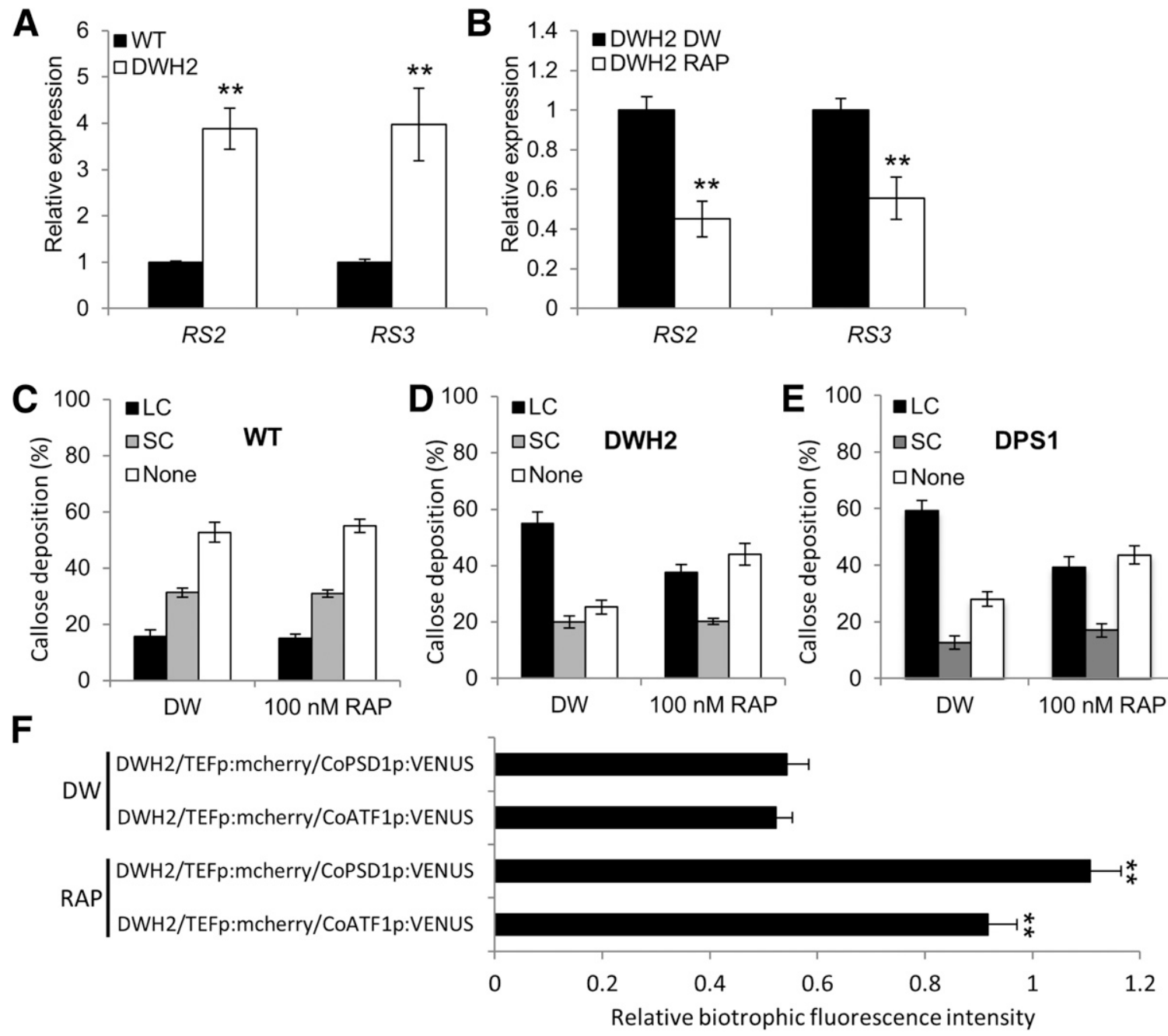

Fig. 10. TOR signaling regulated by CoWhi2 is required for hemibiotrophic infection. A, Expression of $C$. orbiculare RS 2 and $R S 3$ in the Cowhi2 $\Delta$ mutant at $6 \mathrm{~h}$ postinoculation (hpi). Conidial suspensions of each strain were placed on detached cotyledons of cucumber and incubated at $24^{\circ} \mathrm{C}$. Total RNA was extracted from the cotyledons, and expression of CoRS2 and CoRS3 was analyzed by quantitative reverse transcription-polymerase chain reaction (qRT-PCR). Three independent experiments were conducted. Standard errors are indicated. WT $=$ wild-type 104-T and DWH2 $=$ Cowhi2 $\Delta$ mutant. Asterisks represent significant differences between cucumber leaves inoculated with the WT and Cowhi2 $\Delta$ mutant (Student's $t$ test $P<0.01$ ). B, Expression of CoRS2 and CoRS3 in the Cowhi $2 \Delta$ mutant treated with rapamycin using qRT-PCR. RAP $=100 \mathrm{nM}$ rapamycin and DW $=$ distilled water. $\mathbf{C}$ to $\mathbf{E}$, Mean frequency of the callose deposition types beneath appressoria of each strain treated with rapamycin. Approximately 200 appressoria were observed per inoculated site, and three replicates were examined for each of three independent experiments; standard errors are indicated. $100 \mathrm{nM}$ RAP $=100 \mathrm{nM}$ rapamycin, WT $=$ wild-type $104-\mathrm{T}$, DWH $2=$ Cowhi2 $\Delta$ mutant, DPS1 = Copsr1 $\Delta$ mutant. F, Quantification of fluorescence intensity of biotrophy-specific expression of VENUS in the Cowhi2 $\Delta$ mutant at 80 hpi after treatment with rapamycin. At least 50 VENUS fluorescence signals from infection hyphae were measured for each strain. The mean biotrophic fluorescence intensity value was calculated by dividing VENUS fluorescence intensity by mCherry fluorescence intensity in infection hyphae of each strain. Asterisks represent significant differences between Cowhi2 $\Delta$ mutant treated with rapamycin and untreated mutant (Student's $t$ test: $P<0.01$ ). $\mathrm{RAP}=100 \mathrm{nM}$ rapamycin, $\mathrm{DW}=$ distilled water. 
revealed that the activity of the TOR signaling pathway is regulated by CoWhi2 at $4 \mathrm{hpi}$, when the conidial germ tube begins to swell to form the appressorium, leading to the regulation of hemibiotrophic infection necessary for host infection. Therefore, we considered that, in the hemibiotrophs $M$. oryzae and $C$. orbiculare, the highly regulated TOR activity during appressorium formation is essential for successful infection of the host plant. In $M$. oryzae, TOR activation is required for biotrophic growth (Fernandez et al. 2014). Our present analysis did not deal with the TOR signaling pathway during the biotrophic and necrotrophic stages after penetration; therefore, determining the relevance between CoWhi2 and the TOR signaling pathway during these two stages will provide a deeper understanding of the molecular mechanisms of hemibiotrophic infection. Interestingly, the microarray revealed that the top 150 upregulated genes, aside from the many ribosomal protein genes, in the Cowhi2s mutant treated with rapamycin were not downregulated compared with the Cowhi2s mutant without rapamycin treatment, suggesting that CoWhi2 also negatively regulates TOR-independent signal pathways. However, we consider that the biotrophic stage in hemibiotrophic infection in C. orbiculare is largely dependent on TOR rather than being a TOR-independent pathway, based on the plant responses against the Cowhi2s mutant and the biotrophic stage in the Cowhi2s mutant treated with rapamycin. In this study, we thus implicate a Whi2-mediated network in the regulation of hemibiotrophy in C. orbiculare (Fig. 12).
Environmental signal transduction through CoWhi2 and the downstream regulatory cascade that controls the biotrophic stage of hemibiotrophic infection.

What factor does CoWhi2 interact with during initial appressorium development? In $S$. cerevisiae, Whi2p physically interacts with phosphatase Psr1p, and this complex dephosphorylates the transcription factor Msn2p, which has a pivotal role in general stress responses by mediating the transcription of hundreds of genes (Boy-Marcotte et al. 1998; Kaida et al. 2002). In this study, we found that CoWhi2 physically interacts with CoPsr1 and that the phenotype of the Copsr1s mutant is similar to that of the Cowhi2 $\Delta$ mutant. Therefore, we assumed that a CoWhi2-CoPsr1 signaling pathway may contribute to the biotrophic stage of hemibiotrophic infection. In S. cerevisiae, Whi2p-Psr1p has a role as a positive regulator of Msn2p, because Msn2p activity for Msn2p-mediated gene expression under stress conditions is lower in the whi2 $\Delta$ and psr $1 \Delta$ mutant (Kaida et al. 2002; Sadeh et al. 2011). On the other hand, in the TOR pathway, Tor $1 p$ and Tor $2 p$ act as negative regulators of Msn2p in $S$. cerevisiae, because Msn2p activity is elevated in tor $1 \Delta$ and tor $2 \Delta$ mutants (Sadeh et al. 2011). In addition, the activity of the TOR pathway is suppressed in response to nutrient starvation but activated in response to nutrients (Rohde et al. 2008). Thus, Msn2p activity, activated by Whi2-Psr1 signaling transduction and suppression of TOR activity in response to nutrient starvation, significantly induces Msn2p-mediated gene expression. Hemibiotrophic M. oryzae utilizes nutrients produced
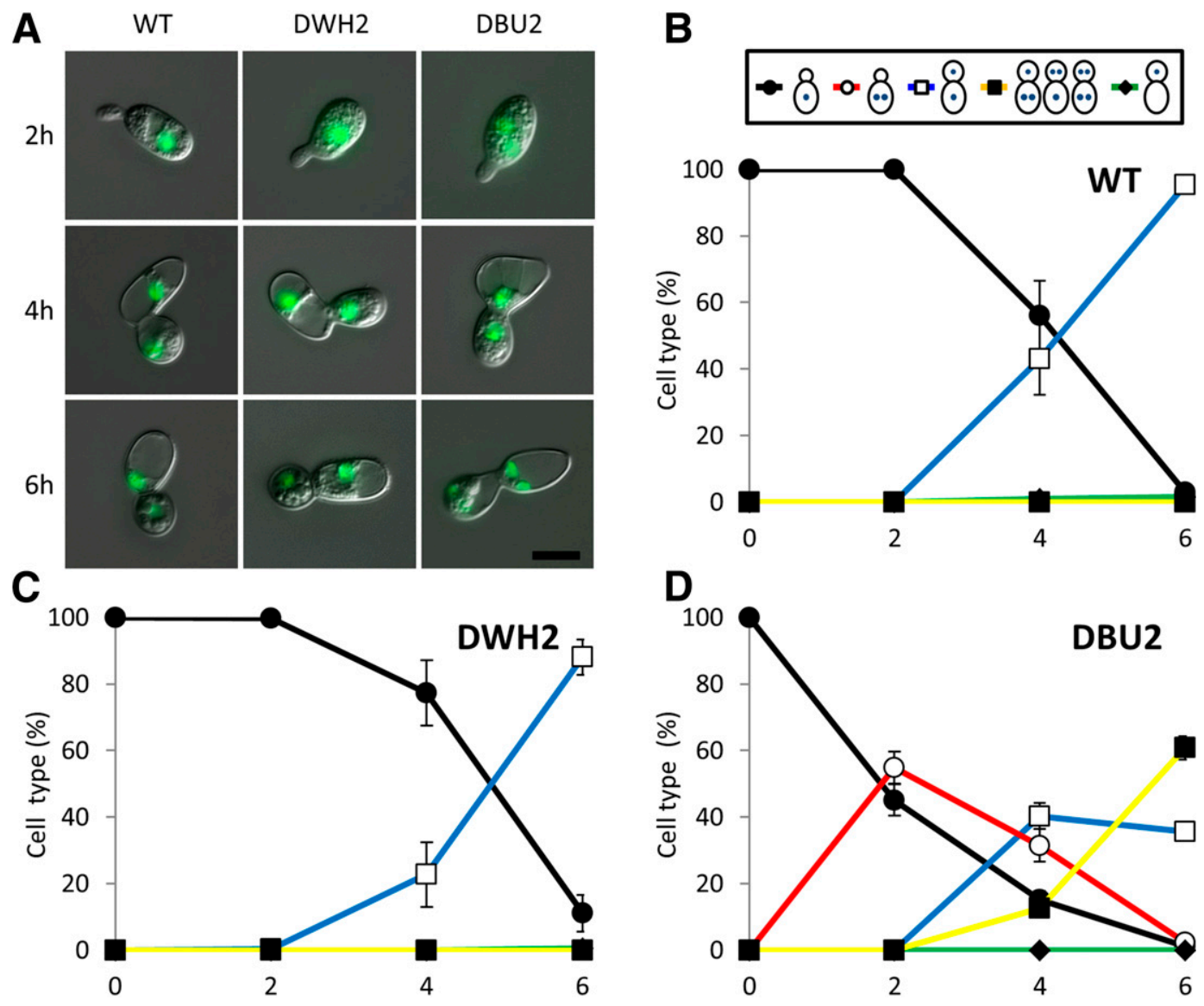

Fig. 11. Nuclear division in the Cowhi2 $\Delta$ mutant during appressorium formation. A, Time-course series of micrographs to show nuclei in conidia and infection structures of the wild type, the Cowhi2 $\Delta$ mutant, and the Cobub2 $\Delta$ mutant during appressorium development. The HISTONE H1-GFP gene fusion vectors were introduced into the three strains. Nuclei (green) were observed during appressorium development. Scale bar $=5 \mu \mathrm{m}$. B to D, Mean percentage $( \pm$ standard error: $n=3$ ) of cells with various patterns of nuclear distribution. Scoring as shown in top diagram: one nucleus retained in the conidium (black), two nuclei retained in the conidium (red), one nucleus in the conidium and the other in the appressorium through mitosis (blue), multiple nuclei in the conidium and appressorium through two or three rounds of mitosis (yellow), and one nucleus in the appressorium and degradation of the remaining nucleus in the conidium through autophagy (green). At least 200 conidia were scored at each timepoint. WT $=$ wild type, DWH2 $=$ Cowhi2 $\Delta$ mutant, and DBU2 $=$ Cobub2 $\Delta$ mutant. 
by autophagy in response to the lack of nutrients on the host plant surface until its infection hyphae can obtain nutrients from host tissues (Deng et al. 2012). In our study, TOR-dependent ribosomal gene expression in the Cowhi2d mutant is significantly upregulated at 4 hpi, suggesting that CoWhi 2 negatively regulates TOR activity when nutrients are unavailable. In M. oryzae, MoMSN2 encodes the $\mathrm{C}_{2} \mathrm{H}_{2}$ zinc-finger DNA-binding domain, the homolog of MSN2 in S. cerevisiae, and is involved in pleiotropic functions, including conidiogenesis, appressorium formation, pathogenicity, and invasive hyphal growth, through Msn2-mediated gene expression (Zhang et al. 2014). Based on $C$. orbiculare genome information, we confirmed that the predicted amino acid sequence of gene ENH88318 in C. orbiculare conserves high similarity to $S$. cerevisiae Msn2p and M. oryzae MoMsn2, particularly in its zinc-finger domain. Moreover, we showed that the Cowhi2s mutant has increased resistance to CFW on PDA, similar to the case of the M. oryzae Momsn2A mutant, indicating that CoWhi2 may likely respond to a stress by regulating CoMsn2-mediated stress-responsive genes (Zhang et al. 2014). Therefore, we speculated that this gene in C. orbiculare acts as an MSN2, which putatively functions in the regulation of various genes necessary for infection. On the basis of our data and previous reports, we assumed that CoWhi2CoPsr1 signal transduction and the inactivation of TOR by CoWhi2 increase activity of $C$. orbiculare CoMsn2, a homolog of Msn2p, which potentially may result in CoMsn2-mediated gene expression necessary for the infection stage during nutrient deprivation.

An important question is the role of CoWHI2 in hemibiotrophic infection by $C$. orbiculare. The TOR pathway in $S$. cerevisiae is activated in response to nutrient homeostasis, leading to synthesis of ribosomal proteins (Rohde et al. 2008). In our study, deletion of CoWHI2 led to upregulation of ribosomal gene expression regulated by TOR activity. Therefore, we assumed that the loss of CoWhi2 function in response to nutrient homeostasis inside host tissues activates TOR. On the basis of our data and previous reports, we hypothesize that the downregulation of CoMsn2 resulting from the loss of the CoWhi2-CoPsr1 signal pathway and from the activation of TOR resulting from the loss of CoWhi2 function inside the nutrient-rich host tissues is a primary contributor as hemibiotrophic infection of $C$. orbiculare (Deng et al. 2012; Sadeh et al. 2011).

In $C$. higginsianum, the biotrophic, bulbous infection hyphae are confined to the initially infected epidermal cell, and development switches to narrower, necrotrophic infection hyphae, which then ramify throughout the host cells (O'Connell et al. 2012). In Colletotrichum graminicola, the biotrophic infection hyphae grow not only in the initially infected cell but also into cells adjacent to the first infected cell; it then switches to necrotrophic infection hyphae, which ramify throughout the host cells (Vargas et al. 2012). Therefore, we assumed that the switch from biotrophy to necrotrophy in Colletotrichum spp. could be

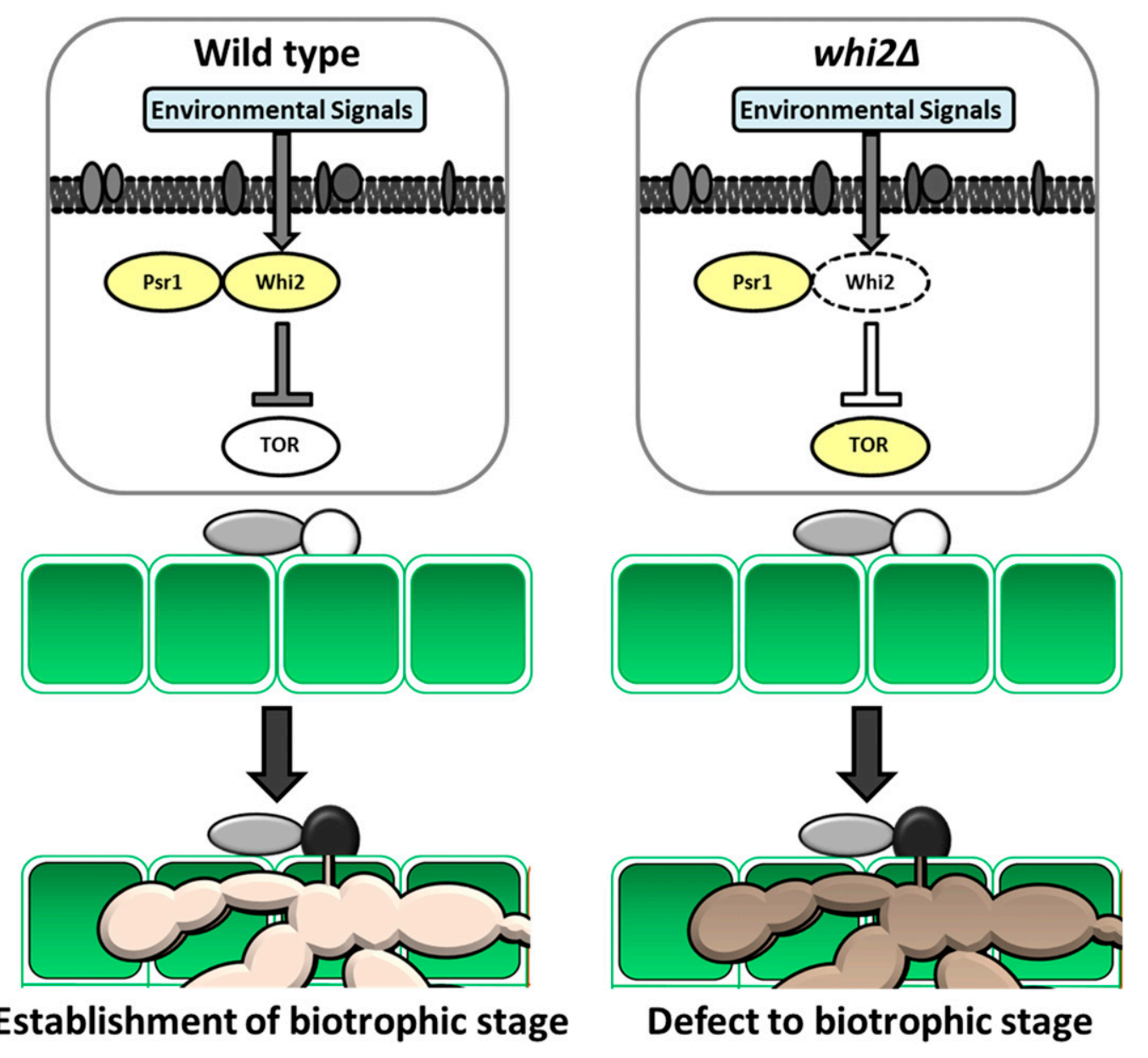

Fig. 12. Model for the Colletotrichum orbiculare Whi2 signal network involved in the biotrophic stage of hemibiotrophic infection of $C$. orbiculare. Environmental signals affect several intracellular events associated with infection-related morphogenesis in $C$. orbiculare. In the $C$. orbiculare wild type, CoWhi2 negatively regulates TOR activity at the time of appressorium development, resulting in establishment of the highly regulated biotrophic stage of hemibiotrophic infection. On the other hand, in the Cowhi2s mutant, TOR is activated at the time of appressorium development, resulting in the disturbance of the establishment of the biotrophic stage of hemibiotrophic infection. 
achieved by sensing a particular condition in the host plant. Our future work will focus on revealing extracellular environmental signals and fungal-sensing mechanisms to control the transition from biotrophic to necrotrophic in $C$. orbiculare for a deeper understanding of hemibiotrophic fungi-host plant interactions.

\section{MATERIALS AND METHODS}

Fungal and bacterial strains, culture conditions, transformation, and genomic DNA blot analysis.

Strain 104-T (MAFF240422) of Colletotrichum orbiculare (Berk. \& Mont.) Arx [syn. C. lagenarium (Pass.); Ellis \& Halst.] was used as the wild type. All C. orbiculare strains were cultured on PDA $(3.9 \%$ [wt/vol] PDA) (Difco Laboratories, Detroit) at $24^{\circ} \mathrm{C}$. For observing vegetative growth under stress conditions, the fungal strains were cultured on PDA containing $0.1 \mathrm{mg}$ of CFW per milliliter, $0.2 \mathrm{mg}$ of Congo red (CR) per milliliter, and $0.005 \%$ SDS. Escherichia coli DH5 $\alpha$-competent cells cultured in Luria-Bertani medium (Sambrook et al. 1989) at $37^{\circ} \mathrm{C}$ were used as the host for gene manipulation. When required, kanamycin was added to the medium at $50 \mu \mathrm{g} / \mathrm{ml}$. Agrobacterium tumefaciens $\mathrm{C} 58 \mathrm{C} 1$ cultured in Luria-Bertani medium at $28^{\circ} \mathrm{C}$ was used to transform $C$. orbiculare based on a previous protocol (Harata and Kubo 2014). Hygromycinresistant transformants were selected on PDA containing $50 \mu \mathrm{g}$ of hygromycin B per milliliter (Wako Chemicals, Osaka, Japan) and $25 \mu \mathrm{g}$ of Meropen per milliliter (Sumitomo Dainippon Pharma, Osaka, Japan). Bialaphos-resistant transformants of C. orbiculare were selected on SD medium containing $1.25 \mu \mathrm{g}$ of bialaphos per milliliter (Meiji Seika Pharma, Tokyo) and $25 \mu \mathrm{g}$ of Meropen per milliliter. Neomycin-resistant transformants were selected on PDA (Merck KGaA, Darmstadt, Germany) containing $50 \mu \mathrm{g}$ of G418 per milliliter (Nacalai Tesque, Kyoto, Japan) and $25 \mu \mathrm{g}$ of Meropen per milliliter. Sulfonylurea-resistant transformants were selected on SD medium containing $4 \mu \mathrm{g}$ of chlorimuron-ethyl per milliliter (Chem Service, West Chester, PA, U.S.A.) and $25 \mu \mathrm{g}$ of Meropen per milliliter. Total DNA extraction from mycelia of $C$. orbiculare and DNA blot analysis were done as previously described (Harata and Kubo 2014).

\section{Pathogenicity tests.}

A pathogenicity assay on cucumber cotyledons (Cucumis sativus L. 'Suyo') was performed as described by Tsuji et al. (1997), using conidia of $C$. orbiculare from 7-day-old cultures. Drops of a conidial suspension $\left(10 \mu \mathrm{l}\right.$ of $5 \times 10^{5}$ conidia per milliliter) were placed at different locations on the surface of detached cucumber cotyledons. After inoculation, the cotyledons were incubated in a humid box at $24^{\circ} \mathrm{C}$ for 5 or 7 days.

\section{Light microscopy.}

For localization analysis of CoRas 2 and histone H1-GFP during appressorium formation, conidia were harvested from 7-day-old PDA cultures and were suspended in distilled water. The conidial suspension, adjusted to $5 \times 10^{5}$ conidia per milliliter, was placed in an eight-well multitest slide (ICN Biomedicals, Aurora, OH, U.S.A.) and was incubated in humid boxes at $24^{\circ} \mathrm{C}$. Red fluorescent protein (RFP)-CoRas2 and histone H1-GFP signals were observed using a Carl ZEISS Imager M2 microscope (Zeiss, Gottingen, Germany), with an excitation/barrier filter set of $470 \mathrm{~nm} / 509 \mathrm{~nm}$ for GFP and $595 \mathrm{~nm} / 620 \mathrm{~nm}$ for RFP. For observing infectious hyphae in cucumber leaves, the abaxial surface of cucumber cotyledons was inoculated with drops of a conidial suspension and was incubated at $24^{\circ} \mathrm{C}$ for 3 days. Then, the epidermal cells at the inoculation site were excised with forceps and were stained with $0.1 \%$ (wt/vol) lactophenol-aniline blue. The stained tissues were observed with a Nikon ECLIPSE E600 microscope (Nikon, Tokyo). For detecting callose deposition in epidermal cells, the abaxial surface of cucumber cotyledons was inoculated with a conidial suspension in distilled water or in water with $100 \mathrm{nM}$ rapamycin or $0.5 \mu \mathrm{M}$ DPI and was incubated at $24^{\circ} \mathrm{C}$ for $24 \mathrm{~h}$. The epidermal cells at the inoculation site were stained with $0.01 \%$ aniline blue in $0.15 \mathrm{M} \mathrm{K}_{2} \mathrm{HPO}_{4}$ and, then, were observed with a Nikon ECLIPSE E600 microscope. For detecting $\mathrm{H}_{2} \mathrm{O}_{2}$ cytochemically, inoculated cucumber cotyledons were stained with a $1 \mathrm{mg} / \mathrm{ml}$ DAB solution, were incubated for $24 \mathrm{~h}$ at room temperature in the dark, and were observed with the Nikon ECLIPSE E600 microscope. For observing VENUS signals under the control of the biotrophy-specific promoter and mCherry signals under the control of the TEF promoter of $C$. orbiculare, the abaxial surface of cucumber cotyledons was inoculated with a conidial suspension with or without $100 \mathrm{nM}$ rapamycin and was incubated at $24^{\circ} \mathrm{C}$ for 72 or $80 \mathrm{~h}$. Then, the epidermal cells at the inoculation site were excised with forceps and were observed with a Carl ZEISS Imager M2 microscope and an excitation barrier set of 470/509 $\mathrm{nm}$ for GFP and $595 / 620 \mathrm{~nm}$ for RFP. To quantify the biotrophic fluorescence intensity in the infection hyphae of each transformant, the images were analyzed using the software ImageJ (National Institutes of Health, Bethesda, MD, U.S.A.).

\section{Vector construction.}

All primers used in this study are listed in Supplementary Table S2. We constructed a CoWHI2 complementation vector pBIG4MRBrev-CoWHI2 as follows. The CoWHI2 gene fragment, including $0.7 \mathrm{~kb}$ upstream of the translation start site of CoWHI2 and $1.3 \mathrm{~kb}$ downstream of the translation termination site of CoWHI2, and the pBIG4MRBrev vector fragment carrying the bialaphos-resistance gene were amplified by PCR, using primer pairs CoWHI2F1A and CoWHI2R1B and pBICoWHI2F1A and pBICoWHI2R1B for the pBIG4MRBrev vector fragment, respectively. Then the pBIG4MRBrev-CoWHI2 vector was constructed using the In-Fusion HD cloning kit (Takara Bio, Otsu, Japan) with the amplified product.

Next, we constructed the gene-replacement vector pBIG4MRBrevcowhi2 as follows. The hygromycin-resistance gene and the pBIG4MRBrev-CoWHI2 vector fragment, not including the region from the translation start site of CoWHI2 to the translation termination site of CoWHI2, were amplified by PCR using primer pairs CoWHI2hphF1A and CoWHI2hphR1B and pBICoWHI2F2A and pBICoWHI2R2B, respectively. Then the pBIG4MRBrev-cowhi2 vector was constructed using the In-Fusion HD cloning kit with the amplified product.

We constructed the CoPSRI gene-replacement vector pBIG4MRSrev-copsr1 as follows. The CoPSR1 gene fragment, including $1.3 \mathrm{~kb}$ upstream of the translation start site of CoPSR1 and $1.1 \mathrm{~kb}$ downstream of the translation termination site of CoPSR 1, and the pBIuescriptSK vector fragment were amplified by PCR using primer pairs CoPSR1F1A and CoPSR1R1B and pBSCoPSR1F1A and pBSCoPSR1R1B, respectively. Then the pBIuescriptSK-CoPSR1 vector was constructed using the InFusion HD cloning kit with the amplified product.

Next, we amplified the neomycin-resistance gene and linearized the pBIuescriptSK-CoPSR1 vector fragment, not including the region from the translation start site of CoPSRI to the translation termination site of COPSR1, by PCR, using primer pairs CoPSR1neoF1A and CoPSR1neoR1B and pBSCoPSR1F2A and pBSCoPSR1R2B, respectively. Then the pBIuescriptSKcopsr1 vector was constructed using the In-Fusion HD cloning kit with the amplified product.

Finally, we amplified the neomycin-resistance fragment, including the upstream region of the translation start site of CoPSR 1 and downstream region of the translation termination 
site of CoPSRl, and the pBIG4MRSrev vector fragment carrying the sulfonylurea-resistance gene by $\mathrm{PCR}$, using primer pairs CoPSR1F2A and CoPSR1R2B and pBIG4MRBSrevF1A and pBIG4MRBSrevR1B, respectively. Then the pBIG4MRSrevcopsr1 vector was constructed using the In-Fusion HD cloning kit with the amplified product.

To assess behavior of biotrophic-specific expression genes in the Cowhi 2 mutant, we constructed the pBICoPSD $1_{\text {pro }}$ :VENUS vector carrying the biotrophy-specific expression VENUS gene by the following methods. The CoPSD1 gene fragment, including $2.4 \mathrm{~kb}$ upstream of the translation start site of CoPSD1 and $0.8 \mathrm{~kb}$ downstream of the translation termination site of CoPSD1, and the pBIG4MRBrev vector fragment were amplified by PCR using primer pairs CoPSD1F1A and CoPSD1R1B and pBIG4MRBSrevF1A and pBIG4MRBSrevR1B, respectively. Then, the pBIG4MRBrev-CoPSD1 vector was constructed, using the In-Fusion HD cloning kit with the amplified product. Next, we amplified the VENUS gene and pBIG4MRBrev-CoPSD1 vector fragment, not including the region from the translation start site of CoPSD1 to the translation termination site of CoPSD1, by PCR using primer pairs VENUSF1A and VENUSR1B and pBICoPSD1F1A and pBICoPSD1R1B, respectively. Then, the pBICoPSD $1_{\text {pro: }}$ VENUS vector was constructed, using the InFusion HD cloning kit with the amplified product.

To analyze the behavior of biotrophic-specific expression genes in the Cowhi $2 \Delta$ mutant, we constructed the pBICoATF $1_{\text {pro: }}$ : VENUS vector carrying the biotrophic-specific expression gene $V E N U S$ as follows. The CoATF 1 gene fragment, including $2.1 \mathrm{~kb}$ upstream of the translation start site of CoATF 1 and $1.5 \mathrm{~kb}$ downstream of the translation termination site of CoATF1, and the pBIG4MRBrev vector fragment were amplified by PCR, using primer pairs CoATF1F1A and CoATF1R1B and pBIG4MRBSrevF1A and pBIG4MRBSrevR1B, respectively. Then the pBIG4MRBrev-CoATF1 vector was constructed using the In-Fusion HD cloning kit with the amplified product.

Next, we amplified the VENUS gene and pBIG4MRBrevCoATF1 vector fragment, not including the region from the translation start site of COATF1 to the translation termination site of CoATF1, by PCR using primer pairs VENUSF1A and VENUSR1B and pBICoATF1F1A and pBICoATF1R1B, respectively. Then the $\mathrm{pBICoATF} 1_{\text {pro }}$ :VENUS vector was constructed using the In-Fusion HD cloning kit with the amplified product.

To analyze the biotrophic fluorescence intensity from infection hyphae of the Cowhi2s mutant, we constructed the pBITEFpro:mCherry vector carrying the constitutive and highly expressed $m$ Cherry gene as follows. The TEF promoter fragment and the pBIG4MRSrev:mCherry vector fragment carrying the sulfonylurea-resistance gene were amplified by PCR, using primer pairs TEFF1A and TEFR1B and pBIG4MRBSrevF1A and mCherryR1B, respectively. Then the pBITEFpro:mCherry vector was constructed, using the In-Fusion HD cloning kit with the amplified product. To visualize nuclear division, histone H1-GFP fusion gene cloned in pBICoHIS1-GFP was introduced into the wild type and Cowhi2s mutant by ATMT (Fukada and Kubo 2015).

\section{qRT-PCR analysis.}

A conidial suspension $\left(10 \mu \mathrm{l}\right.$ of $1.0 \times 10^{6}$ conidia per milliliter) in distilled water or $100 \mathrm{nM}$ rapamycin was placed on the surface of a detached cucumber cotyledon at different locations and was incubated in a humid box at $24^{\circ} \mathrm{C}$. At $6,12,24,48$, and 72 hpi, total RNA was extracted from the cotyledons inoculated with the respective strains, using a Qiagen RNeasy plant mini kit (Qiagen) and was treated with RNase-free DNase kit (Qiagen). qRT-PCR was performed using the primers listed in Supplementary Table S3. Expression of actin genes in Cucumber sativus and $C$. orbiculare was used as a control (Tanaka et al. 2007) for normalizing the level of mRNA. The Power SYBR Green RNA-to CT 1-step kit was used for all qRT-PCR tests (Life Technologies, Carlsbad, CA, U.S.A.). Quantitative analysis of each mRNA was performed using the Applied Biosystems StepOne System (Life Technologies).

\section{Yeast two-hybrid interaction assays.}

The yeast two-hybrid screen was performed following the instructions of the Matchmaker Gold yeast two-hybrid system (Takara Bio). Full-length cDNAs of putative interaction partners were generated from 3 -day-old $C$. orbiculare mycelia. The genes encoding the proteins tested for interaction were cloned into pGBKT7 or pGADT7 vectors (Takara Bio), to express fusion proteins with the yeast GAL4 binding (BD) and activation domain (AD), respectively. All $\mathrm{BD}$ or $\mathrm{AD}$ constructs were transformed into the Gold or Y187 yeast strain (Takara Bio). After mating, diploid yeasts were plated on double dropout synthetic selective medium lacking Trp and Leu (DDO) for mating control, on stringent medium supplemented with $20 \mu \mathrm{g}$ of $\mathrm{X}-\alpha-\mathrm{Gal}$ and $100 \mathrm{ng}$ of aureobasidin A per milliliter (DDO/X/AbA) and four-dropout synthetic selective medium lacking Trp, Leu, His, and Ade (QDO) andv were incubated at $28^{\circ} \mathrm{C}$ for 5 days. Growth of all diploid yeasts carrying both pGADT7-AD- and pGBKT7-BD-transformed vectors on DDO was examined. Protein interactions were assessed by the growth of diploid yeasts on DDO, $\mathrm{DDO} / \mathrm{X} / \mathrm{AbA}$, and QDO media, compared with diploid yeasts carrying pGBKT7-Lam and pGADT7-T, used as a negative control.

\section{Microarray analysis.}

Microarray analyses were performed using the Colletotrichum orbiculare $(8 \times 60,000,13,352$ independent probes, Design ID: 060762) oligo microarray, according to the Agilent 60-mer Oligo Microarray Processing Protocol (Agilent Technologies). Total RNA samples (200 ng) were used to prepare Cy3-labeled cRNA, using the Low RNA Input fluorescent linear amplification kit (Agilent Technologies). Fluorescence-labeled cRNAs were purified using an RNeasy RNA purification kit (Qiagen, Hilden, Germany). Three independent RNA samples were used to confirm the reproducibility of the microarray analyses. The images were analyzed using Feature Extraction Software (ver. 10.7.3.1) and GeneSpring GX 12.1 software (Agilent Technologies). Normalization was performed as follows: i) intensity-dependent Lowess normalization; ii) data transformation, with measurements set to $\leq 0.01$; iii) per chip: 75 th percentile normalization of each array; and iv) per gene: Data were normalized to control samples (wild type). The top 150 upregulated genes in Cowhi2d compared with wild type were selected and were used for the hierarchical clustering (heat map) analysis. The raw and processed data were deposited in the Gene Expression Omnibus (GEO) database (accession number GSE73096).

\section{ACKNOWLEDGMENTS}

We thank K. Shirasu and P. Gan for providing genome information of C. orbiculare for the microarray analysis. We thank B. E. Hazen for carefully reading the article and giving valuable suggestions. This work was supported by The Japan Society for the Promotion of Science KAKENHI grant numbers 24248009 and $15 \mathrm{H} 05780$.

\section{LITERATURE CITED}

Alers, S., Löffler, A. S., Wesselborg, S., and Stork, B. 2012. Role of AMPKmTOR-Ulk1/2 in the regulation of autophagy: Cross talk, shortcuts, and feedbacks. Mol. Cell. Biol. 32:2-11.

Bonawitz, N. D., Chatenay-Lapointe, M., Pan, Y., and Shadel, G. S. 2007. Reduced TOR signaling extends chronological life span via increased respiration and upregulation of mitochondrial gene expression. Cell Metab. 5:265-277. 
Boy-Marcotte, E., Perrot, M., Bussereau, F., Boucherie, H., and Jacquet, M. 1998. Msn2p and Msn4p control a large number of genes induced at the diauxic transition which are repressed by cyclic AMP in Saccharomyces cerevisiae. J. Bacteriol. 180:1044-1052.

Brown, E. J., Albers, M. W., Shin, T. B., Ichikawa, K., Keith, C. T., Lane W. S., and Schreiber, S. L. 1994. A mammalian protein targeted by G1arresting rapamycin-receptor complex. Nature 369:756-758.

Chen, D., Wang, Y., Zhou, X., Wang, Y., and Xu, J. R. 2014. The Sch9 kinase regulates conidium size, stress responses, and pathogenesis in Fusarium graminearum. PLoS One 9:e105811.

Chi, M. H., Park, S. Y., Kim, S., and Lee, Y. H. 2009. A novel pathogenicity gene is required in the rice blast fungus to suppress the basal defenses of the host. PLoS Pathog. 5:e1000401.

Crespo, J. L., and Hall, M. N. 2002. Elucidating TOR signaling and rapamycin action: Lessons from Saccharomyces cerevisiae. Microbiol. Mol. Biol. Rev. 66:579-591.

Deng, Y., Qu, Z., and Naqvi, N. I. 2012. Role of macroautophagy in nutrient homeostasis during fungal development and pathogenesis. Cells 1:449-463.

Fernandez, J., Marroquin-Guzman, M., and Wilson, R. A. 2014. Evidence for a transketolase-mediated metabolic checkpoint governing biotrophic growth in rice cells by the blast fungus Magnaporthe oryzae. PLoS Pathog. 10:e1004354.

Fujikawa, T., Sakaguchi, A., Nishizawa, Y., Kouzai, Y., Minami, E., Yano, S., Koga, H., Meshi, T., and Nishimura, M. 2012. Surface $\alpha-1,3$-glucan facilitates fungal stealth infection by interfering with innate immunity in plants. PLoS Pathog. 8:e1002882.

Fukada, F., and Kubo, Y. 2015. Colletotrichum orbiculare regulates cell cycle G1/S progression via a two-component GAP and a GTPase to establish plant infection. Plant Cell 27:2530-2544.

Gan, P., Ikeda, K., Irieda, H., Narusaka, M., O’Connell, R. J., Narusaka, Y., Takano, Y., Kubo, Y., and Shirasu, K. 2013. Comparative genomic and transcriptomic analyses reveal the hemibiotrophic stage shift of Colletotrichum fungi. New Phytol. 197:1236-1249.

Glazebrook, J. 2005. Contrasting mechanisms of defense against biotrophic and necrotrophic pathogens. Annu. Rev. Phytopathol. 43:205-227.

Görner, W., Durchschlag, E., Martinez-Pastor, M. T., Estruch, F., Ammerer, G., Hamilton, B., Ruis, H., and Schüller, C. 1998. Nuclear localization of the $\mathrm{C} 2 \mathrm{H} 2$ zinc finger protein Msn2p is regulated by stress and protein kinase A activity. Genes Dev. 12:586-597.

Harata, K., and Kubo, Y. 2014. Ras GTPase activating protein CoIra1 is involved in infection-related morphogenesis by regulating cAMP and MAPK signaling pathways through CoRas2 in Colletotrichum orbiculare. PLoS One 9:e109045.

Heitman, J., Movva, N. R., and Hall, M. N. 1991. Targets for cell cycle arrest by the immunosuppressant rapamycin in yeast. Science 253:905-909.

Irieda, H., Maeda, H., Akiyama, K., Hagiwara, A., Saitoh, H., Uemura, A., Terauchi, R., and Takano, Y. 2014. Colletotrichum orbiculare secretes virulence effectors to a biotrophic interface at the primary hyphal neck via exocytosis coupled with SEC22-mediated traffic. Plant Cell 26: 2265-2281.

Kaida, D., Yashiroda, H., Toh-e, A., and Kikuchi, Y. 2002. Yeast Whi2 and Psr1-phosphatase form a complex and regulate STRE-mediated gene expression. Genes Cells 7:543-552.

Kleemann, J., Rincon-Rivera, L. J., Takahara, H., Neumann, U., Ver Loren van Themaat, E., van der Does, H. C., Hacquard, S., Stüber, K., Will, I., Schmalenbach, W., Schmelzer, E., and O'Connell, R. J. 2012. Sequential delivery of host-induced virulence effectors by appressoria and intracellular hyphae of the phytopathogen Colletotrichum higginsianum. PLoS Pathog. 8:e1002643.

Kubo, Y., and Furusawa, I. 1991. Melanin biosynthesis: Prerequisite for successful invasion of the plant host by appressoria of Colletotrichum and Pyricularia. Pages 205-218 in: The Fungal Spore and Disease Initiation in Plants and Animals. G. T. Cole, and H. C. Hoch, eds. Plenum Publishing, New York.

Kubo, Y., and Takano, Y. 2013. Dynamics of infection-related morphogenesis and pathogenesis in Colletotrichum orbiculare. J. Gen. Plant Pathol. 79:233-242.

Kunz, J., Henriquez, R., Schneider, U., Deuter-Reinhard, M., Movva, N. R., and Hall, M. N. 1993. Target of rapamycin in yeast, TOR2, is an essential phosphatidylinositol kinase homolog required for G1 progression. Cell 73:585-596.

Lanver, D., Mendoza-Mendoza, A., Brachmann, A., and Kahmann, R. 2010. Sho1 and Msb2-related proteins regulate appressorium development in the smut fungus Ustilago maydis. Plant Cell 22: 2085-2101.

Latgé, J. P. 2010. Tasting the fungal cell wall. Cell. Microbiol. 12:863-872.

Leadsham, J. E., Miller, K., Ayscough, K. R., Colombo, S., Martegani, E. Sudbery, P., and Gourlay, C. W. 2009. Whi2p links nutritional sensing to actin-dependent Ras-cAMP-PKA regulation and apoptosis in yeast. J. Cell Sci. 122:706-715

Liu, W., Zhou, X., Li, G., Li, L., Kong, L., Wang, C., Zhang, H., and Xu, J. R. 2011. Multiple plant surface signals are sensed by different mechanisms in the rice blast fungus for appressorium formation. PLoS Pathog. 7:e1001261.

Loewith, R., and Hall, M. N. 2011. Target of rapamycin (TOR) in nutrient signaling and growth control. Genetics 189:1177-1201.

López-Berges, M. S., Rispail, N., Prados-Rosales, R. C., and Di Pietro, A. 2010. A nitrogen response pathway regulates virulence functions in Fusarium oxysporum via the protein kinase TOR and the bZIP protein MeaB. Plant Cell 22:2459-2475.

Marroquin-Guzman, M., and Wilson, R. A. 2015. GATA-dependent glutaminolysis drives appressorium formation in Magnaporthe oryzae by suppressing TOR inhibition of cAMP/PKA signaling. PLoS Pathog. 11:e1004851.

Martin, D. E., Powers, T., and Hall, M. N. 2006. Regulation of ribosome biogenesis: Where is TOR? Cell Metab. 4:259-260.

Martin, D. E., Soulard, A., and Hall, M. N. 2004. TOR regulates ribosomal protein gene expression via PKA and the Forkhead transcription factor FHL1. Cell 119:969-979.

Martínez-Pastor, M. T., Marchler, G., Schüller, C., Marchler-Bauer, A., Ruis, H., and Estruch, F. 1996. The Saccharomyces cerevisiae zinc finger proteins Msn2p and Msn4p are required for transcriptional induction through the stress response element (STRE). EMBO J. 15:2227-2235.

Mentlak, T. A., Kombrink, A., Shinya, T., Ryder, L. S., Otomo, I., Saitoh, H., Terauchi, R., Nishizawa, Y., Shibuya, N., Thomma, B. P., and Talbot, N. J. 2012. Effector-mediated suppression of chitin-triggered immunity by magnaporthe oryzae is necessary for rice blast disease. Plant Cell 24 322-335.

Miya, A., Albert, P., Shinya, T., Desaki, Y., Ichimura, K., Shirasu, K., Narusaka, Y., Kawakami, N., Kaku, H., and Shibuya, N. 2007. CERK1, a LysM receptor kinase, is essential for chitin elicitor signaling in Arabidopsis. Proc. Natl. Acad. Sci. U.S.A. 104:19613-19618.

Monaghan, J., and Zipfel, C. 2012. Plant pattern recognition receptor complexes at the plasma membrane. Curr. Opin. Plant Biol. 15 349-357.

Nicholson, R. L., and Hammerschmidt, R. 1992. Phenolic compounds and their role in disease resistance. Annu. Rev. Phytopathol. 30:369-389.

O’Connell, R.J., Thon, M.R., Hacquard, S., Amyotte, S.G., Kleemann, J., Torres, M.F., Damm, U., Buiate, E.A., Epstein, L., Alkan, N., Altmüller, J., Alvarado-Balderrama, L., Bauser, C.A., Becker, C., Birren, B.W., Chen, Z., Choi, J., Crouch, J.A., Duvick, J.P., Farman, M.A., Gan, P., Heiman, D., Henrissat, B., Howard, R. J., Kabbage, M., Koch, C., Kracher, B., Kubo, Y., Law, A. D., Lebrun, M. H., Lee, Y.-H., Miyara, I., Moore, N., Neumann, U., Nordström, K., Panaccione, D. G., Panstruga, R., Place, M., Proctor, R. H., Prusky, D., Rech, G., Reinhardt, R., Rollins, J. A., Rounsley, S., Schardl, C. L., Schwartz, D. C., Shenoy, N., Shirasu, K., Sikhakolli, U. R., Stüber, K., Sukno, S. A., Sweigard, J. A., Takano, Y., Takahara, H., Trail, F., van der Does, H. C., Voll, L. M., Will, I., Young, S. Zeng, Q., Zhang, J., Zhou, S., Dickman, M. B., Schulze-Lefert, P., Ver Loren van Themaat, E., Ma, L.-J., and Vaillancourt, L. J. 2012. Lifestyle transitions in plant pathogenic Colletotrichum fungi deciphered by genome deciphered by genome and transcriptome analyses. Nat. Genet. 44:1060-1065.

Penninckx, I. A., Thomma, B. P., Buchala, A., Métraux, J. P., and Broekaert, W. F. 1998. Concomitant activation of jasmonate and ethylene response pathways is required for induction of a plant defensin gene in Arabidopsis. Plant Cell 10:2103-2113.

Perfect, S. E., O’Connell, R. J., Green, E. F., Doering-Saad, C., and Green, J. R. 1998. Expression cloning of a fungal proline-rich glycoprotein specific to the biotrophic interface formed in the Colletotrichum-bean interaction. Plant J. 15:273-279.

Radcliffe, P., Trevethick, J., Tyers, M., and Sudbery, P. 1997. Deregulation of CLN1 and CLN2 in the Saccharomyces cerevisiae whi2 mutant. Yeast 13:707-715.

Richmond, T. 2000. Higher plant cellulose synthases. Genome Biol. 1: reviews3001.1-reviews3001.6.

Rohde, J. R., Bastidas, R., Puria, R., and Cardenas, M. E. 2008. Nutritional control via Tor signaling in Saccharomyces cerevisiae. Curr. Opin. Microbiol. 11:153-160.

Rossi, F. R., Gárriz, A., Marina, M., Romero, F. M., Gonzalez, M. E., Collado, I. G., and Pieckenstain, F. L. 2011. The sesquiterpene botrydial produced by Botrytis cinerea induces the hypersensitive response on plant tissues and its action is modulated by salicylic acid and jasmonic acid signaling. Mol. Plant-Microbe Interact 24:888-896.

Sabatini, D. M., Erdjument-Bromage, H., Lui, M., Tempst, P., and Snyder, S. H 1994. RAFT1: A mammalian protein that binds to FKBP12 in a rapamycindependent fashion and is homologous to yeast TORs. Cell 78:35-43. 
Sadeh, A., Movshovich, N., Volokh, M., Gheber, L., and Aharoni, A. 2011. Fine-tuning of the Msn2/4-mediated yeast stress responses as revealed by systematic deletion of Msn2/4 partners. Mol. Biol. Cell 22: 3127-3138.

Saitoh, H., Fujisawa, S., Mitsuoka, C., Ito, A., Hirabuchi, A., Ikeda, K., Irieda, H., Yoshino, K., Yoshida, K., Matsumura, H., Tosa, Y., Win, J., Kamoun, S., Takano, Y., and Terauchi, R. 2012. Large-scale gene disruption in Magnaporthe oryzae identifies MC69, a secreted protein required for infection by monocot and dicot fungal pathogens. PLoS Pathog. 8:e1002711.

Sambrook, J., Fritsch, E. F., and Maniatis, T. 1989. Molecular Cloning: A Laboratory Manual. Cold Spring Harbor Laboratory Press, Cold Spring Harbor, NY, U.S.A

Spoel, S. H., Johnson, J. S., and Dong, X. 2007. Regulation of tradeoffs between plant defenses against pathogens with different lifestyles. Proc. Natl. Acad. Sci. U.S.A. 104:18842-18847.

Su'udi, M., Kim, M. G., Park, S. R., Hwang, D. J., Bae, S. C., and Ahn, I. P. 2011. Arabidopsis cell death in compatible and incompatible interactions with Alternaria brassicicola. Mol. Cells 31:593-601.

Tanaka, S., Ishihama, N., Yoshioka, H., Huser, A., O’Connell, R., Tsuji, G., Tsuge, S., and Kubo, Y. 2009. The Colletotrichum orbiculare SSD1 mutant enhances Nicotiana benthamiana basal resistance by activating a mitogen-activated protein kinase pathway. Plant Cell 21: 2517-2526.

Tanaka, S., Yamada, K., Yabumoto, K., Fujii, S., Huser, A., Tsuji, G., Koga, H., Dohi, K., Mori, M., Shiraishi, T., O'Connell, R., and Kubo, Y. 2007. Saccharomyces cerevisiae SSD1 orthologues are essential for host infection by the ascomycete plant pathogens Colletotrichum lagenarium and Magnaporthe grisea. Mol. Microbiol. 64:1332-1349.

Torres, M. A., Jones, J. D., and Dangl, J. L. 2005. Pathogen-induced, NADPH oxidase-derived reactive oxygen intermediates suppress spread of cell death in Arabidopsis thaliana. Nat. Genet. 37:1130-1134.

Tsuji, G., Takeda, T., Furusawa, I., Horino, O., and Kubo, Y. 1997. Carpropamid, an anti-rice blast fungicide, inhibits scytalone dehydratase activity and appressorial penetration in Colletotrichum lagenarium. Pestic. Biochem. Physiol. 57:211-219.
Vargas, W. A., Martín, J. M., Rech, G. E., Rivera, L. P., Benito, E. P., DíazMínguez, J. M., Thon, M. R., and Sukno, S. A. 2012. Plant defense mechanisms are activated during biotrophic and necrotrophic development of Colletotricum graminicola in maize. Plant Physiol. 158:1342-1358.

Verma, D. P., and Hong, Z. 2001. Plant callose synthase complexes. Plant Mol. Biol. 47:693-701.

Williams, B., Kabbage, M., Kim, H. J., Britt, R., and Dickman, M. B. 2011. Tipping the balance: Sclerotinia sclerotiorum secreted oxalic acid suppresses host defenses by manipulating the host redox environment. PLoS Pathog. 7:e1002107.

Wilson, R. A., and Talbot, N. J. 2009. Under pressure: Investigating the biology of plant infection by Magnaporthe oryzae. Nat. Rev. Microbiol. 7:185-195.

Yoshino, K., Irieda, H., Sugimoto, F., Yoshioka, H., Okuno, T., and Takano, Y. 2012. Cell death of Nicotiana benthamiana is induced by secreted protein NIS1 of Colletotrichum orbiculare and is suppressed by a homologue of $C g D N 3$. Mol. Plant-Microbe Interact 25: 625-636.

Yoshioka, H., Numata, N., Nakajima, K., Katou, S., Kawakita, K., Rowland, O., Jones, J. D., and Doke, N. 2003. Nicotiana benthamiana gp91 ${ }^{\text {phox }}$ homologs NbrbohA and NbrbohB participate in $\mathrm{H}_{2} \mathrm{O}_{2}$ accumulation and resistance to Phytophthora infestans. Plant Cell 15:706-718.

Yu, F., Gu, Q., Yun, Y., Yin, Y., Xu, J. R., Shim, W. B., and Ma, Z. 2014. The TOR signaling pathway regulates vegetative development and virulence in Fusarium graminearum. New Phytol. 203:219-232.

Zhang, H., Zhao, Q., Guo, X., Guo, M., Qi, Z., Tang, W., Dong, Y., Ye, W., Zheng, X., Wang, P., and Zhang, Z. 2014. Pleiotropic function of the putative zinc-finger protein MoMsn2 in Magnaporthe oryzae. Mol. Plant-Microbe Interact 27:446-460.

Zhang, S., and Xu, J. R. 2014. Effectors and effector delivery in Magnaporthe oryzae. PLoS Pathog. 10:e1003826.

\section{AUTHOR-RECOMMENDED INTERNET RESOURCE}

National Institutes of Health ImageJ softare: rsb.info.nih.gov/ij 